\title{
SUR CERTAINS ESPACES FIBRÉS PRINCIPAUX DIFFÉRENTIABLES ET HOLOMORPHES
}

\author{
SHINGO MURAKAMI
}

Le but principal de ce mémoire est d'étudier les espaces fibrés principaux différentiables et holomorphes de groupe abelien connexe ayant pour base une $C$-variété au sens de Wang [12]. Une $C$-variété $X$ étant une variété complexe compacte simplement connexe et homogène, $X$ est la base d'un espace fibré principal qui est un groupe de Lie où opère un sous-groupe fermé par les translations à droite comme groupe structural. Pour cette raison, on considère d'abord dans le $\S 1$ les espaces fibrés principaux différentiables de groupe abelien connexe $A$ ayant pour base la base $X$ d'un certain espace fibré principal différentiable $Y$ de groupe $B$. Parmi ces espaces fibrés principaux les plus simples sont ceux qui sont associés à $Y$ par les homomorphismes du groupe $B$ dans le groupe $A$. On montre que tous les espaces fibrés principaux différentiables qui sont trivialisés par la projection de $Y$ sur $X$ sont de ce type si $Y$ est simplement connexe et si $B$ est un groupe compact. Dans le $\S 2$, on donne une classification des espaces fibrés principaux dont la base est une $C$-variété $X$ et dont le groupe est un groupe de Lie complexe abelien connexe $A$ : Tout espace fibré principal différentiable de base $X$ et de groupe $A$ est sous-jacent à un espace fibré principal holomorphe et, de plus, quand on pose d'après Wang $X=G / U$ où $G$ est un groupe de Lie complexe semi-simple simplement connexe, tout espace fibré holomorphe du type en question est un espace fibré associé à l'espace fibré $G$ de base $X$ et de groupe $U$ par un homomorphisme holomorphe bien déterminé du groupe $U$ dans le groupe $A$. On étudie enfin, dans le $\S 3$, les connexions définies sur les espaces fibrés principaux holomorphes de groupe abelien connexe ayant pour base une $C$-variété. On y montre en particulier qu'un tel espace fibré principal est trivial si il admet une connexion holomorphe.

Je tiens à exprimer ici toute ma reconnaissance à M. J.-L. Koszul, dont

Received May 1, 1959. 
les nombreuses suggestions m'ont grandement aidé et m'ont permis d'améliorer considérablement la rédaction de ce mémoire.

§1. Espaces fibrés principaux différentiables de groupe abelien associés à un espace fibré principal.

1. Dans ce paragraphe, on désignera par $A$ un groupe de Lie abelien connexe. Soit $X$ une variété différentiable. On sait que l'ensemble des classes d'espaces fibrés principaux différentiables de base $X$ et de groupe $A$ peut être identifié avec le premier groupe de cohomologie $H^{1}\left(X, \mathbf{A}_{d}\right)$ de $X$ (à support fermé) à coefficients dans le faisceau $\mathbf{A}_{d}$ des germes de fonctions différentiables sur $X$ à valeurs dans $A$ [6]. Supposons maintenant que $X$ est la base d'un espace fibré principal différentiable $Y$ de groupe $B, B$ étant un groupe de Lie quelconque. La projection de $Y$ sur $X$ sera notée $q$ et les opérations de $B$ dans $Y$ seront écrites à droite. Alors la projection $g: Y \rightarrow X$ induit un homomorphisme $\rho$ du groupe $H^{1}\left(X, \mathbf{A}_{d}\right)$ dans $H^{1}\left(Y, \mathbf{A}_{d}\right)$. Le noyau de $\rho$ n'est autre que l'ensemble des classes d'espaces fibrés principaux de base $X$ et de groupe $A$ qui sont trivialisés par $q: Y \rightarrow X$. D'autre part, les homomorphismes différentiables du groupe $B$ dans le groupe $A$ forment un groupe abelien $\operatorname{Hom}(B, A)$ de manière évidente. En faisant correspondre à un homomorphisme $f: B \rightarrow A$ la classe des espaces fibrés $P$ associés à $Y$ par $f$, on a une application $\theta$ de Hom $(B, A)$ dans $H^{1}\left(X, \mathbf{A}_{d}\right)$. On voit que $\theta$ est un homomorphisme du groupe Hom $(B, A)$ dans le groupe $H^{1}\left(X, \mathbf{A}_{d}\right)$. En effet, si $Y$ est défini par le système de fonctions de passage $\left\{g_{L v}\right\}$ relatif à un recouvrement ouvert $\{U, V, \ldots\}$ de $X, P$ est défini par le système de fonctions de passage $f\left(g_{U V}\right)$ relatif au même recouvrement ouvert de $X$.

THÉORÈME 1. Soit $Y$ un espace fibré principal différentiable de base $X$ et de groupe $B$. Supposons que $Y$ soit simplement connexe et que $B$ soit compact. Alors, la suite

$$
(0) \longrightarrow \operatorname{Hom}(B, A) \stackrel{\theta}{\longrightarrow} H^{1}\left(X, \mathbf{A}_{d}\right) \stackrel{\rho}{\longrightarrow} H^{1}\left(Y, \mathbf{A}_{d}\right)
$$

est exacte.

En particulier, sous les mêmes hypothèses du théorème, tout espace fibré principal différentiable $P$ de base $X$ et de groupe $A$ trivialisé par la projection $q: Y \rightarrow X$ est un espace fibré associé à $Y$ par un homomorphisme du groupe 
$B$ dans le groupe $A$ qui est bien déterminé par $P$.

Corollaire 1. Avec les mêmes conditions que dans le théorème, si $H^{2}(Y, \mathrm{Z})$ $=(0)$ pour le faisceau constant $\mathrm{Z}$ dont le groupe est le groupe des nombres entiers, $\theta$ est bijectif.

En effet, puisque le revêtement universel de $A$ est isomorphe à un espace vectoriel réel $R^{n}$, il existe une suite exacte

$$
(0) \rightarrow \Pi \rightarrow R^{n} \rightarrow A \rightarrow(0),
$$

où $\Pi$ est un sous-groupe discret de $R^{n}$. Designant par $\Pi$ le faisceau constant sur $Y$ de groupe $\Pi$, on a alors la suite exacte de faisceaux sur $Y$

$$
(0) \rightarrow \Pi \rightarrow \mathbf{R}_{d}^{n} \rightarrow \mathbf{A}_{d} \rightarrow(0),
$$

et cela definit la suite exacte de cohomologie

$$
\cdots \rightarrow H^{1}\left(Y, \mathbf{R}_{d}^{n}\right) \rightarrow H^{1}\left(Y, \mathbf{A}_{d}\right) \rightarrow H^{2}(Y, \Pi) \rightarrow \cdots .
$$

Puisque tout espace fibré principal de groupe $R^{n}$ est trivial, on a $H^{1}\left(Y, \mathbf{R}_{d}^{\prime \prime}\right)$ $=(0)$. D'autre part, puisque $\Pi$ est un groupe abelien libre de rang fini, le faisceau $\Pi$ est somme directe des faisceaux isomorphes à $\mathbf{Z}$. Par conséquent, d'après l'hypothèse du corollaire, $H^{2}(Y, \Pi)=(0)$. La suite exacte ci dessus implique alors que $H^{1}\left(Y, \mathbf{A}_{d}\right)=(0)$ et le corollaire résulte donc de la suite exacte du théorème.

Comme on sait, si $K$ est un groupe de Lie compact simplement connexe, $H^{2}(K, \mathbf{Z})=(0)$. Par conséquent, d'après le corollaire 1 , on a le

Corollaire 2. Soit $K$ un groupe de Lie compact simplement connexe et soit $B$ un sous-groupe fermé de $K$. L'homomorphisme $\theta$ de $\operatorname{Hom}(B, A)$ dans $H^{1}\left(K / B, \mathbf{A}_{d}\right)$, qui est défini par la structure d'espace fibré principal différentiable de base $K / B$ et de groupe $B$ sur $K$, est bijectif.

2. Espaces fibrés principaux trivialisés par la projection d'un espace fibré principal. On conserve les notations du $\S 1.1$. Pour la démonstration $d u$ Théorème 1 ainsi qu'en vue des paragraphes suivants, on considère ici les espaces fibrés principaux différentiables de groupe $A$ qui sont trivialisés par la projection $q: Y \rightarrow X$. Rappelons d'abord qu'un facteur sur $Y$ à valeurs dans $A$ est une fonction différentiable $k(y, s)$ sur le produit $Y \times B$ à valeurs dans $A$ qui vérifie la condition 


$$
k(y, s) k(y s, t)=k(y, s t)
$$

quels que soient $y \in Y$ et $s, t \in B$, et que deux facteurs $k(y, s), k^{\prime}(y, s)$ sont dits équivalents si il existe une fonction différentiable $r$ sur $Y$ à valeurs dans $A$ telle que

$$
k^{\prime}(y, s)=r(y) k(y, s) r(y s)^{-1}
$$

quels que soient $y \in Y, s \in B$. On sait qu'il existe une correspondance bijective entre l'ensemble des classes d'équivalence de facteurs sur $Y$ à valeurs dans $A$ et l'ensemble des classes d'espaces fibrés principaux différentiables de base $X$ et de groupe $A$ qui sont trivialisés par la projection $q: Y \rightarrow X$ [7]. En fait, étant donné un espace fibré principal différentiable $P$ de base $X$ et de groupe $A$ trivialisé par $q: Y \rightarrow X$, tout facteur $k$ correspondant à la classe de $P$ est défini, au moyen d'une application différentiable $q^{*}: Y \rightarrow P$ qui est compatible avec $q$ (c'est-à-dire, qui vérifie la condition $p q^{*}=q, p$ étant la projection de $P$ sur $X$ ), par la formule

$$
q^{*}(y s)=q^{*}(y) k(y, s)
$$

pour $y \in Y, s \in B$. Par ailleurs, un facteurs $k(y, s)$ sur $Y$ à valeurs dans $A$ qui ne dépend que de $s$ peut être identifié avec un homomorphisme différentiable $f$ du groupe $B$ dans $A$, les deux étant liés par la relation $f(s)=k(y, s)$ pour $s \in B$; un espace fibré $P$ dont la classe correspond à la classe de ce facteur est un espace fibré associé à $Y$ par l'homomorphisme $f: B \rightarrow A$ [8]. Il en résulte que, pour que cet espace fibré $P$ soit trivial, il faut et il suffit qu'il existe une fonction différentiable $r$ sur $Y$ à valeurs dans $A$ telle que

$$
f(s)=r(y) r(y s)^{-1}
$$

quels que soient $y \in Y, s \in B$. On remarque que les notions introduites ici pour les espaces fibrés différentiables sont également définies pour les espaces fibrés holomorphes quand on y remplace les mots "différentiable" et "groupe de Lie" par "holomorphe" et "groupe de Lie complexe" respectivement.

Soit $P$ un espace fibré principal différentiable de base $X$ et de groupe $A$; la projection de $P$ sur $X$ sera notée $p$. Supposons que $P$ est trivialisé par la projection $q: Y \rightarrow X$ et qu'un facteur $k$ est défini par $(1,1)$ au moyen d'une application $q^{*}: Y \rightarrow P$ compatible avec $q$. Soit $\omega$ une forme de connexion sur l'espace fibré $P$; c'est une forme de degré 1 sur $P$ à valeurs dans l'algèbre dẹ 
Lie a du groupe $A .^{1{ }^{12)}}$ On sait que la forme $\eta=\omega q^{*}$, image réciproque de $\omega$ par $q^{*}: Y \rightarrow P$, est alors une forme sur $Y$ à valeurs dans a vérifiant les deux conditions suivantes.

$$
\begin{aligned}
& \eta((d y) s)-\eta(d y)=k(y, s)^{-1} k(d y, s), \\
& \eta(y d s)=k(y, s)^{-1} k(y, d s)
\end{aligned}
$$

quels que soient $y \in Y, s \in B$, où $d y$ (resp. $d s$ ) désigne un vecteur tangent quelconque d'origine $y \in Y$ (resp. d'origine $s \in B$ ). Réciproquement, si une forme $\eta$ sur $Y$ à valeurs dans a vérifie ces deux conditions, il existe une forme de connexion $\omega$ sur $P$ telle que $\eta=\omega q^{*}$ [8]. D'autre part, puisque $A$ est un groupe abelien, la forme $d \omega$ est l'image réciproque $\Omega p$ d'une forme $\Omega$ de degré 2 sur $X$ ̀̀ valeurs dans a par la projection $p: P \rightarrow X ; \Omega$ est la forme de courbure sur $X$ de la connexion définie par $\omega$. On a alors $\Omega q=\Omega p q^{*}=(d(\omega)) q^{*}=d\left(\omega q^{*}\right)$ et par suite

$$
\Omega q=d \eta
$$

Supposons en particulier que $P$ est un espace fibré associé à $Y$ par un homomorphisme $f: B \rightarrow A$; il existe donc une application $q^{*}: Y \rightarrow P$ compatible avec $q$ qui définit le facteur $k(y, s)=f(s)(y \in Y, s \in B)$ par la formule $(1,1)$. Soit $\gamma$ une forme de connexion sur l'espace fibré principal $Y$; c'est une forme de degré 1 sur $Y$ à valeurs dans l'algèbre de Lie $\mathfrak{b}$ du groupe $B$ qui vérifie les conditions $\gamma((d y) s)=\operatorname{ad}\left(s^{-1}\right) \gamma(d y)$ et $\gamma(y d s)=s^{-1} d s$ quels que soient $y \in Y$ et $s \in B$. La forme $\Gamma=d \gamma+[\gamma, \gamma]$ est la forme de courbure sur $Y$ de la connexion définie par $\gamma$. Désignant encore par $f$ l'homomorphisme de l'algèbre de Lie $\mathfrak{b}$ dans l'algèbre de Lie $a$ induit par $f: B \rightarrow A$, on considère la forme $\eta=f(\gamma)$ sur $Y$ à valeurs dans $a$. On a $\eta((d y) s)=f(\gamma((d y) s))=f\left(\operatorname{ad}\left(s^{-1}\right) \gamma(d y)\right)=f(\gamma(d y))$ $=\eta(d y)$, puisque a est abelienne; d'autre part, $\eta(y d s)=f(\gamma(y d s))=f\left(s^{-1} d s\right)$ $=f(s)^{-1} f(d s)$. Par conséquent, la forme $\eta$ vérifie les conditions $(1,2)$ et $(1,3)$. D'après ce qu'on a rappelé plus haut, il existe donc une forme de connexion $\omega$ sur $P$ telle que $\eta=\omega q^{*}$. La connexion de l'espace fibré principal $P$ définie par $\omega$ sera dite la connexion de $P$ canoniquement associée à la connexion $r d e Y$. Par ailleurs, puisque $f$ est un homomorphisme de $\mathfrak{b}$ dans l'algèbre de Lie

1) Pour les notions concernant les connexions, voir, par exemple, [11].

2) Dans ce mémoire, l'algèbre de Lie d'un groupe de Lie $G$ est canoniquement identifiée avec l'espace tangent à $G$ en l'élément neutre de $G$. 
abelienne a, on a $f(\Gamma)=f(d \gamma)=d(f(\gamma))$. . D'après $(1,4)$, la forme de courbure $\Omega$ sur $X$ de la connexion $\omega$ vérifie donc la relation suivante.

$$
\begin{aligned}
\Omega q & =f(\Gamma) \\
& =f(d \gamma) .
\end{aligned}
$$

3. Démonstration du Théorème 1. On montre d'abord que l'homomorphisme $\theta$ est injectif. Supposons qu'un élément $f \in \operatorname{Hom}(B, A)$ appartienne au noyau de $\theta$; cela signifie qu'un espace fibré $P$ associé à $Y$ par l'homomorphisme $f: B \rightarrow A$ est trivial. D'après ce qu'on a vu au $\S 1.2$, il existe alors une fonction différentiable $r$ sur $Y$ à valeurs dans $A$ telle que

$$
f(s)=r(y) r(y s)^{-1}
$$

quels que soient $y \in Y, s \in B$. Puisque $Y$ est simplement connexe, il existe une fonction différentiable $\widetilde{r}$ sur $Y$ à valeurs dans le revêtement universel $R^{n}$ de $A$ telle que $r=\pi \widetilde{r}, \pi$ étant la projection de $R^{n}$ sur $A$. On a alors

$$
f(s)=\pi(\tilde{r}(y)-\tilde{r}(y s))
$$

quels que soient $y \in Y, s \in B$. Pour chaque élément $s \in B$, l'image réciproque de $f(s)$ par $\pi$ est discrète dans $R^{n}$. Puisque $Y$ est connexe, il en résulte que $\tilde{r}(y)-\tilde{r}(y s)$ ne dépend que de $s$; on peut donc poser

$$
\widetilde{f}(s)=\widetilde{r}(y)-\tilde{r}(y s)
$$

quels que soient $y \in Y, s \in B$. Alors, si $s, t \in B, \tilde{f}(s t)=(\widetilde{r}(y)-\tilde{r}(y s))+(\tilde{r}(y s)$ $-\widetilde{r}(y s t))=\widetilde{f}(s)+\widetilde{f}(t)$, ce qui montre que $\tilde{f}$ est un homomorphisme du groupe $B$ dans le groupe $R^{n}$. Le groupe $B$ étant compact, l'homomorphisme $\tilde{f}$ est trivial. Puisque $f(s)=\pi(\tilde{f}(s))$ pour $s \in B$, l'homomorphisme $f: B \rightarrow A$ est donc trivial, ce qui démontre l'injectivité de $\theta$.

Il reste à montrer que l'image de $\theta$ coïncide avec le noyau de $\rho$. D'après les définitions de $\theta$ et de $\rho$, il suffit de démontrer qu'un espace fibré principal $P$ de base $X$ et de groupe $A$ trivialisé par $q: Y \rightarrow X$ est un espace fibré associé à $Y$ par un homomorphisme du groupe $B$ dans le groupe $A$. On choisit une forme de connexion $\omega$ sur $P$. Soit $\Omega$ la forme de courbure sur $X$ de la connexion $\omega$. D'après $(1,4)$, on a $\Omega q=d \eta$, où $\eta$ est l'image réciproque $\omega q^{*}$ de la forme $\omega$ par une application $q^{*}: Y \rightarrow P$ compatible avec $q$. Puisque la forme $\Omega q$ est invariante par les opérations du groupe compact $B$ dans $Y$, il existe 
alors une forme $\eta_{1}$ invariante par $B$ sur $Y$, ̀̀ valeurs dans a, telle que $\Omega q=d \eta_{1}$ [5]. La.forme $\zeta=\eta_{1}-\eta$ est une forme fermée sur $Y$ à valeurs dans $a ; d \zeta=0$. Puisque $Y$ est simplement connexe, la forme $\zeta$ est donc la différentielle $d \tilde{r}$ d'une fonction différentiable $\widetilde{r}$ sur $Y$ à valeurs dans a. Posons $r(y)=\exp (\widetilde{r}(y))$ pour $y \in Y$, exp étant l'application exponentielle de $\mathfrak{a}$ dans $A$. La fonction différentiable $r$ sur $Y$ à valeurs dans $A$ ainsi définie vérifie la relation

$$
r(y)^{-1} r(d y)=\zeta(d y)
$$

quel que soit $y \in Y$. On définit maintenant une application $q_{1}^{*}: Y \rightarrow P$ compatible avec $q$ en posant:

$$
q_{1}^{*}(y)=q^{*}(y) r(y)
$$

pour $y \subseteq Y$. Alors, $\left(\omega q_{1}^{*}\right)(d y)=\omega\left(q^{*}(d y) r(y)+q^{*}(y) r(d y)\right)=\eta(d y)+\zeta(d y)$ $=\eta_{1}(d y)$, ce qui montre que $\eta_{1}=\omega q_{1}^{*}$. Un facteur $k(y, s)$ correspondant à la classe de $P$ étant défini au moyen de l'application $q_{1}^{*}: Y \rightarrow P$ par la formule $(1,1)$, ce facteur $k(y, s)$ et la forme $\eta_{1}$ vérifient les conditions $(1,2)$ et $(1,3)$. Puisque la forme $\eta_{1}$ est invariante par $B$, il résulte de $(1,2)$ que $k(d y, s)=0$ quels que soient $y \in Y$ et $s \in B$. Comme $Y^{\prime}$ est connexe, cela démontre que le facteur $k(y, s)$ ne dépend que de $s$; par conséquent, $P$ est un espace fibré associé à $Y$ par l'homomorphisme $f: B \rightarrow A$ défini par $f(s)=k(y, s)$. Le Théorème 1 est ainsi démontré.

§2. Espaces fibrés principaux holomorphes de groupe abelien ayant pour base une $C$-variété

1. Dans les paragraphes suivants $A$ désignera un groupe de Lie complexe abelien connexe. Le revêtement universel de $A$ est isomorphe, comme groupe de Lie complexe, à un espace vectoriel complexe $C^{m}$ de dimension $m$. On a donc la suite exacte

$$
(0) \longrightarrow I \stackrel{\iota}{\longrightarrow} C^{m} \stackrel{\pi}{\rightarrow} A \longrightarrow(0),
$$

où $\Pi$ est un sous-groupe discret de $C^{m}$ et où : est l'injection de $\Pi$ dans $C^{m}$; $\pi: C^{n} \rightarrow A$ est un homomorphisme holomorphe de $C^{m}$ sur $A$.

Soit $X$ une variété complexe. On désignera par $\mathbf{A}_{d}$ (resp. $\mathbf{A}_{h}$ ) le faisceau des germes de fonctions différentiables (resp. holomorphes) sur $X$ à valeurs dans $A$. On a les homomorphismes canoniques de faisceaux $\mu: \mathbf{A}_{h} \rightarrow \mathbf{A}_{d}$ et $\widetilde{\mu}: \mathbf{C}_{h}^{\prime n} \rightarrow \mathbf{C}_{d}^{n}$. Désignons par $\Pi$ lè faisceau constant de groupe $\Pi$ sur $X$. La 
suite exacte $(2,1)$ et les homomorphismes $\iota, \pi$ définissent le diagramme commutatif

$$
\begin{aligned}
& (0) \longrightarrow \Pi \stackrel{\iota h}{\longrightarrow} \mathbf{C}_{h}^{m} \stackrel{\pi h}{\longrightarrow} \mathbf{A}_{h} \longrightarrow(0) \\
& \downarrow \text { id } \quad \sqrt{\mu} \quad \downarrow \mu \\
& (0) \longrightarrow \Pi \stackrel{\iota d}{\longrightarrow} \mathbf{C}_{d}^{m} \stackrel{\pi_{l}}{\longrightarrow} \mathbf{A}_{d} \longrightarrow(0),
\end{aligned}
$$

où les deux lignes sont des suites exactes. (On désigne par id l'application identique). Ce diagramme implique alors le diagramme commutatif suivant concernant les groupes de cohomologie de $X$ (à support fermé) à coefficients dans ces faisceaux.

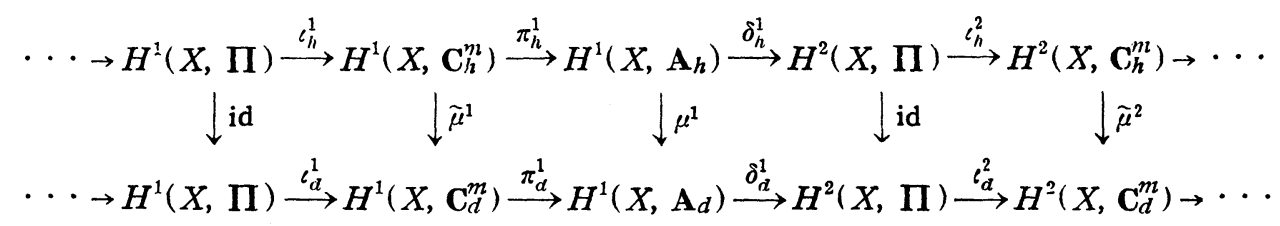

Les deux lignes de ce diagramme sont des suites exactes. Or, on sait que le faisceau $\mathbf{C}_{d}^{m}$ est fin et que le groupe $H^{r}\left(X, \mathbf{C}_{d}^{m}\right)$ est donc nul pour tout entier $r>0$ [6]. D'après le diagramme, il en résulte que $\delta_{d}^{1}$ est bijectif; par conséquent, le noyau de $\mu^{1}$ coïncide avec le noyau de $\delta_{h}^{1}$ et l'image de $\mu^{1}$ coïncide avec le noyau de $\iota_{h}^{2} \delta_{d}^{1}$. Supposons que $X$ soit simplement connexe. Puisque $\Pi$ est un groupe abelien libre de rang fini, on a alors $H^{1}(X, \Pi)=(0)$ et $\pi_{h}^{1}$ est donc injectif. On a ainsi le lemme suivant.

Lemme 1. Si $X$ est une variété complexe simplement connexe, la suite

$$
(0) \longrightarrow H^{1}\left(X, \mathbf{C}_{h}^{m}\right) \stackrel{\pi_{h}^{1}}{\longrightarrow} H^{1}\left(X, \mathbf{A}_{h}\right) \stackrel{\mu^{1}}{\longrightarrow} H^{1}\left(X, \mathbf{A}_{d}\right) \stackrel{\iota_{h}^{2} \delta_{d}^{1}}{\longrightarrow} H^{2}\left(X, \mathbf{C}_{h}^{m}\right)
$$

est exacte.

Quand on identifie le groupe $H^{1}\left(X, \mathbf{A}_{l}\right)$ (resp. $H^{1}\left(X, \mathbf{A}_{d}\right)$ ) avec l'ensemble des classes d'espaces fibrés principaux holomorphes (resp. différentiables) de base $X$ et de groupe $A$, l'homomorphisme $\mu^{1}$ est induit par l'application qui fait correspondre à un espace fibré principal holomorphe $P$ l'espace fibré différentiable sous-jacent à $P$; de même, l'homomorphisme $\pi_{h}^{1}$ est défini par l'application qui fait correspondre à chaque espace fibré principal holomorphe $P$ de base $X$ et de groupe $C^{m}$ la classe des espaces fibrés de groupe $A$ associé à $\dot{P}$ 
par l'homomorphisme $\pi: C^{m t} \rightarrow A$.

Une $C$-variété $X$ est une variété complexe compacte simplement connexe sur laquelle le groupe des homéomorphismes holomorphes de $X$ opère de manière transitive [12].

ThÉoRÈme 2. Soit X une C-variété. Alors, la suite

$$
(0) \longrightarrow H^{1}\left(X, \mathbf{C}_{h}^{n}\right) \stackrel{\pi_{h}^{1}}{\longrightarrow} H^{1}\left(X, \mathbf{A}_{h}\right) \stackrel{\mu^{1}}{\longrightarrow} H^{1}\left(X, \mathbf{A}_{d}\right) \longrightarrow(0)
$$

est exacte.

Corollaire. Si $X$ est une C-variété kaehlerienne, alors l'homomorphisme $\mu^{1}$ de $H^{1}\left(X, \mathbf{A}_{h}\right)$ dans $H^{1}\left(X, \mathbf{A}_{d}\right)$ est bijectif.

Le Théorème 2 et son corollaire impliquent en particulier que tout espace fibré principal différentiable $P$ de groupe $A$ ayant pour base une $C$-variéte $X$ est sous-jacent à un espace fibré principal holomorphe de base $X$ et de groupe $A$ et que, si $X$ est kaehlérienne, la structure de ce dernier est bien déterminée par $P$.

Le corollaire résulte immédiatement du Théorème 2; en effet, si $X$ est une variété compacte kaehlérienne simplement connexe, $\operatorname{dim} H^{1}\left(X, \mathbf{C}_{h}^{n}\right)$ $=\frac{1}{2} \operatorname{dim} H^{1}\left(X, \mathbf{C}^{m}\right)=0$ où $\mathbf{C}^{m}$ désigne le faisceau constant de groupe $C^{m}$ sur $X$ [6]. Remarquons que le corollaire résulte plus directement d'un résultat de Borel-Horzebruch et de Bott [3]; d'après eux, $H^{r}\left(X, \mathbf{C}_{h}^{m}\right)=0$ pour $r>0$ et le Lemme 1 implique que $\mu_{I}$ est bijectif.

Dans la suite, on designera toujours par $X$ une $C$-variéte. On sait que le plus grand groupe connexe Aut $(X)^{\circ}$ des homéomorphismes holomorphes de $X$ muni de la topologie compacts-ouverts est un groupe de Lie complexe réductif qui opère holomorphiquement sur $X$ de manière transitive. Puisque un sousgroupe de Lie semi-simple maximal $G^{\prime}(X)$ de Aut $(X)^{\circ}$ est alors le sous-groupe des commutateurs de Aut $(X)^{\circ}, G^{\prime}(X)$ est un groupe de Lie déterminé par $X$. Le groupe $G^{\prime}(X)$ opère transitivement sur $X$. Soit $G(X)$ le revêtement universel de $G^{\prime}(X)$. Alors, le groupe $G(X)$ est un groupe de Lie complexe semi-simple, déterminé par $X$, qui opère holomorphiquement sur $X$ de manière transitive et presque effective [12].

Soit plus généralement $G$ un groupe de Lie complexe semi-simple simplement connexe qui opère holomorphiquement sur $X$ de manière transitive et 
presque effective. Choisissons un point $x_{0} \in X$. Soit $U$ le sous-groupe de stabilité de $G$ en ce point $x_{0} ; U$ est un sous-groupe de Lie complexe fermé de $G$. L'application $q$ de $G$ sur $X$ définie par

$$
q(y)=y x_{0} \quad \text { pour } \quad y \in G
$$

et les translations à droite de $G$ par les éléments de $U$ définissent sur $G$ une structure d'espace fibré principal holomorphe de base $X$ de groupe $U$ et de projection $q$. Comme au $\$ 1.1$, on a alors l'homomorphisme $\theta_{h}$ du groupe $\operatorname{Hom}_{h}(U, A)$ des homomorphismes holomorphes de $U$ dans $A$ dans le groupe $H^{1}\left(X, \mathbf{A}_{h}\right) ; \theta_{h}$ fait correspondre à un élément $F \in \operatorname{Hom}_{h}(U, A)$ la classe des espaces fibés holomorphes associés à l'espace fibré $G$ par $F: U \rightarrow A$.

THÉRÈme 3. Soit $X$ une C-varieté et soit $G$ un groupe de Lie complexe semi-simple simplement connexe qui opère holomorphiquement sur $X$ de maniere transitive et presque effective. Soit $U$ le sous-groupe de stabilite de $G$ en un point $x_{0} \in X$; on considère $G$ comme espace fibré principal holomorphe de base $X$ et de groupe $U$ opérant par les translations à droite. Alors, l'homomorphisme $\theta_{h}$ du groupe $\operatorname{Hom}_{h}(U, A)$ dans le groupe $H^{1}\left(X, \mathbf{A}_{h}\right)$ est bijectif.

Si l'on prend pour $G$ le groupe $G(X)$ défini ci-dessus, l'énoncé du Théorème 3 concerne uniquement la variété $X$. Par ailleurs, ce théorème dit que tout espace fibré principal holomorphe $P$ de base $X$ et de groupe $A$ est associé à l'espace fibré $G$ par un homomorphisme de $U$ dans $A$ bien déterminé par $P$.

Les Théorèmes 2 et 3 seront démontrés au $\S 2.3$

2. Quelques conséquences des résultats de Wang. Soient $X, G, U, x_{0}, q$ comme dans la section précédente. Soit $K$ un sous-groupe compact maximal de $G$; c'est un groupe de Lie compact semi-simple simplement connexe. Il résulte d'un théorème de Montgomery [9] que le sous-groupe $K$ de $G$ opère transitivement sur $X$; le groupe $U$ étant le sous-groupe de stabilité de $G$ en le point $x_{0} \in X, B=U \cap K$ est le sous-groupe de stabilité de $K$ en ce point $x_{0}$. Puisque $X$ est simplement connexe, il en résulte que les groupes $U$ et $B$ sont simplement connexes. Par ailleurs, l'application $q: G \rightarrow X$ et sa restriction à $K$ induisent les identifications canoniques entre les variétés complexes $X, G / U$ et la variété réelle $K / B$. Pour simplifier l'écriture, on exprime cette situation par la formule 


$$
X=G / U=K / B \text {. }
$$

L'algèbre de Lie $g$ de $G$ est une algèbre de Lie complexe semi-simple." Étant donnée une sous-algèbre de Cartan $\mathfrak{h}$ de $\mathfrak{g}$, le système de racines de $\mathfrak{g}$ par rapport à $\mathfrak{h}$ sera noté $S$, et la décomposition de Cartan de $\mathfrak{g}$ par rapport à $\mathfrak{h}$

$$
\mathfrak{b}=\mathfrak{h}+\sum_{\alpha \in S}\left\{\boldsymbol{e}_{\alpha}\right\}^{(4)}
$$

sera toujours prise de la manière que l'espace réel

$$
\mathfrak{l}=\mathfrak{h}_{k}+\sum_{\alpha \in S}\left\{\boldsymbol{u}_{\alpha}\right\}^{r}+\sum_{\alpha \in S}\left\{v_{\star}\right\}^{r},
$$

où $\mathfrak{h}_{k}=\{h ; h \in \mathfrak{h}, \quad \alpha(h)=-\alpha(h)$ pour tout $\alpha \in S\}$ et où $u_{\alpha}=e_{\alpha}+e_{-\alpha}$, $v_{\alpha}=i\left(e_{\alpha}-e_{-\alpha}\right)$, soit une algèbre de Lie semi-simple compacte. Dans ce cas, le sous-groupe $K$ de $G$ qui correspond à $\mathfrak{i} \subset g$ est un sous-groupe compact maximal de $G$.

D'après Wang [12], par un choix convenable d'une sous-algèbre de Cartan $\mathfrak{h}$ et d'une décomposition de Cartan par rapport à $\mathfrak{h}$, la situation de la sousalgèbre $\mathfrak{u}$ de $\mathfrak{g}$ définie par $U$ est représentée à la façon suivante. Il existe une partition du système de racines $S$ en trois parties disjointes $S_{0}, S_{+}, S_{-}$avec les propriétés suivantes: (i) si $\alpha, \beta \in S_{0}$ et si $\alpha+\beta \in S$, alors $\alpha+\beta \in S_{0}$, (ii) si $\alpha \in S_{0} \cup S_{+}, \beta \in S_{+}$et si $\alpha+\beta \in S$, alors $\alpha+\beta \in S_{+}$et (iii) $\alpha \in S_{+}$si et seulement si $-\alpha \in S_{-} .^{5}$ Soit $\mathfrak{h}_{s}$ le sous-espace complexe de $\mathfrak{h}$ engendré par $h_{\alpha}=\left[e_{\alpha}, e_{-\alpha}\right]$ avec $\alpha \in S_{0}$ et soit $\mathfrak{h}_{0}=\left\{h ; h \in \mathfrak{h}, \alpha(h)=0\right.$ pour toute $\left.\alpha \in S_{0}\right\} ; \mathfrak{h}$ est la somme directe $\mathfrak{h}_{s}+\mathfrak{h}_{0}$. Il existe de plus une décomposition de $\mathfrak{h}_{0}$ en somme directe de deux espaces rationnels (intersections des hyperplans définis par des racines)

$$
\mathfrak{h}_{0}=\mathfrak{h}_{z}+\mathfrak{h}_{w}, \quad \mathfrak{h}_{z} \cap \mathfrak{h}_{w}=(0),
$$

et un sous-espace complexe $\mathfrak{w} \subset \mathfrak{h}_{w}$ tel que

$$
\mathfrak{h}_{w}=\mathfrak{w}+\overline{\mathfrak{w}}, \quad \mathfrak{w} \cap \overline{\mathfrak{w}}=(0),
$$

où - signifie la conjugaison de $g$ par rapport à $¥$. Cela donné, on a :

3) On utilisera, dans la suite, des résultats fondamentaux dans la théorie des groupe de Lie et des algèbres de Lie; pour cela, voir [13] par exemple.

4) $\mathrm{Si} \mathfrak{m} \subset \mathfrak{g}, \mathfrak{m}^{c}\left(\right.$ resp. $\left.\mathfrak{m}^{r}\right)$ désigne le sous-espace complexe (resp. réel) engendré par ill dans $g$.

5) Pour une telle partition de $S$, il existe un ordre linéaire dans $S$ tel que $S_{+}=\{\alpha ; \alpha>0$, $\left.\alpha \notin S_{0}\right\}$ et que si $\alpha \in S_{+}$alors $\alpha>\beta$ pour toute $\beta \in S_{0}$; cela se démontre de la même manière qu'un lemme de Koszul (Sur la forme canonique des espaces homogènes complexes, Canadian J. Math. 7 (1955), pp. 562-576, Lemme 2). Il en résulte qu'il existe une base fondamentale de $S$ dont une partie forme une base fondamentale de $S_{0}$ (cf. I. Satake, On a theorem of E. Cartan, J. Math. Soc. Japan 2 (1951), pp. 284-305). 


$$
\mathfrak{u}=\mathfrak{h}_{s}+\mathfrak{h}_{z}+\mathfrak{w}+\sum_{\alpha \in S_{0}}\left\{e_{\alpha}\right\}^{c}+\sum_{\alpha \in s_{-}}\left\{e_{\alpha}\right\}^{c} .
$$

Soit $₹$ la sous-algèbre de Lie réelle définie par $(2,3)$ utilisant la décomposition de Cartan ci-dessus, et soit $K$ le sous-groupe compact maximal de $G$ ayant $\ddagger$ pour algèbre de Lie. Le sous-groupe compact $B=K \cap U$ correspond à la sous-algèbre $\mathfrak{b}=\mathfrak{l} \cap \mathfrak{u}$. Le sous-espace complexe $\mathfrak{b}^{c}$ de $\mathfrak{g}$ engendré par $\mathfrak{b}$ est une sous-algèbre de Lie complexe de 8 . Il résulte de $(2,4)$ que

$$
\mathfrak{b}^{c}=\mathfrak{h}_{s}+\mathfrak{h}_{z}+\sum_{\alpha \in S_{0}}\left\{\boldsymbol{e}_{\alpha}\right\}^{c} .
$$

L'algèbre de Lie complexe $\mathfrak{b}^{c}$ admet l'algèbre de Lie $\mathfrak{b}$ du groupe compact $B$ comme forme réelle et est donc réductive; le centre de $\mathfrak{b}^{c}$ est $\mathfrak{h}_{z}$, et la composante semi-simple $\mathfrak{b}_{s}^{c}$, qui coïncide avec la sous-algèbre des commutateurs de $\mathfrak{b}^{c}$, est $\mathfrak{h}_{s}+\sum_{\alpha \in s_{0}}\left\{\boldsymbol{e}_{\alpha}\right\}^{c}$. On a :

$$
\mathfrak{b}^{c}=\mathfrak{b}_{s}^{c}+\mathfrak{h}_{z}, \quad \mathfrak{b}_{s}^{c} \cap \mathfrak{h}_{z}=(0) .
$$

Posons

$$
\mathfrak{n}=\sum_{\alpha \in S_{-}}\left\{\boldsymbol{e}_{\alpha}\right\}^{c}
$$

D'après $(2,4)$ et $(2,5), \mathfrak{u}$ est alors la somme directe des sous-espaces

$$
\mathfrak{u}=\mathfrak{b}^{c}+\mathfrak{w}+\mathfrak{n} .
$$

D'après les propriétés de la partition de $S$, il en rèsulte que $\mathfrak{n}$ est un idéal nilpotent de $\mathfrak{u}^{6}{ }^{6}$ On montre que $\mathfrak{b}^{c}+\mathfrak{w}$ coïncide avec le centralisateur de $\mathfrak{h}_{z}+\mathfrak{w}$ dans $\mathfrak{u}$. En effet, il est evident que $\mathfrak{h}_{z}+\mathfrak{w}$ est dans le centre de $\mathfrak{b}^{c}+\mathfrak{w}$. En vertu de $(2,7)$, il suffit donc de montrer que si $n \in \mathfrak{n}$ et si $n \neq 0$ il existe un élément $h \in \mathfrak{h}_{z}+\mathfrak{w}$ tel que $[h, n] \neq 0$. S'il en était pas ainsi, il existerait une racine $\alpha \in S$ - telle que $\alpha(h)=0$ pour tout $h \in \mathfrak{h}_{z}+\mathfrak{w}$, puisque $\left[h, e_{\alpha}\right]=\alpha(h) e_{\alpha}$ pour $h \in \mathfrak{h}$ et $\alpha \in S_{-}$. Puisque les valeurs de $\alpha$ dans $h \cap k$ sont purement imaginaires, $\alpha(\bar{h})=-\overline{\alpha(h)}$. Par conséquent, $\alpha$ serait aussi nulle dans $\bar{h}_{z}+\overline{\mathfrak{m}}$, et donc dans $\mathfrak{h}_{0}=\mathfrak{h}_{z}+\mathfrak{w}+\overline{\mathfrak{w}}$. On sait ${ }^{6)}$ qu'une racine $\alpha$ telle que $\alpha(h)=0$ quel que soit $h \in \mathfrak{h}_{0}$ appartient à $S_{0}$, ce qui contredit $\alpha \in S_{-}$. Cette démonstration montre en même temps que $\left[\mathfrak{h}_{z}+\mathfrak{w}, \mathfrak{n}\right]=\mathfrak{n}$. Par le même raisonnement, on voit que le centre de $\mathfrak{u}$ est nul. Soit d'autre part $\mathfrak{u}^{\prime}$ la sous-algèbre des commutateurs de $\mathfrak{n}$. Il résulte de $(2,7)$ que $\mathfrak{u}^{\prime} \subset \mathfrak{b}_{s}^{c}+\mathfrak{n}$, puisque $\mathfrak{n}$ est un idéal de $\mathfrak{u}$. D'autre part, $\mathfrak{u}^{\prime} \supset\left[\mathfrak{h}_{z}+\mathfrak{w}, \mathfrak{n}\right]=\mathfrak{n}$, et $\mathfrak{u}^{\prime} \supset\left[\mathfrak{b}_{s}^{c}, \mathfrak{b}_{s}^{c}\right]=\mathfrak{b}_{s}^{c}$ puisque l'algèbre de Lie $\mathfrak{b}_{s}^{c}$

6) Cela résulte de ce qu'on a remarqué à la note 5). 
est semi-simple. On a donc:

$$
\mathfrak{n}^{\prime}=\mathfrak{b}_{s}^{c}+\mathfrak{n}
$$

Considérons maintenant le groupe $U$; les sous-algèbres de $\mathfrak{u}$ étant désignées par les lettres gotiques, on désignera par les lettre majuscules correspondantes les sous-groupes de Lie connexes de $U$ engendrés par ces sous-algèbres de Lie. Les sous-groupes $B^{c}, W, N, H_{z}, B^{c} W, H_{z} W$ sont fermés dans $U$. Cela résulte d'un lemme de Wang [12] qui dit qu'un sous-groupe de Lie $L$ est fermé dans $G$ si le normalisateur de la sous-algèbre de Lie $\mathfrak{l}$ définie par $L$ contient $\mathfrak{h}$ et si $\mathfrak{l} \cap \mathfrak{h}_{k}$ engendre un sous-groupe fermé dans $H$. Or, puisque $\mathfrak{b}^{c}+\mathfrak{w}$ cö̈ncide avec le centralisateur de $\mathfrak{h}_{z}+\mathfrak{w}$ dans $\mathfrak{u}, B^{c} W$ est la composante connexe de l'élément neutre $e$ du centralisateur de $H_{z} W$ dans $U$. D'autre part, $N$ est un sous-groupe invariant nilpotent et simplement connexe de $U$. En effet, l'image de $N$ dans le groupe adjoint de $U$ est localement isomorphe à $N$, puisque le centre de $\mathfrak{u}$ est nul ; cette image est, par rapport à une base convenablement choisie de $\mathfrak{u}$, représentée par un groupe des matrices triangulaires dont les élément diagonaux sont tous 1 et est donc un groupe simplement connexe. Par conséquent, le même est vrai pour $N$. Il en résulte en particulier que l'application exponentielle, exp, de $\mathfrak{u}$ dans $U$ induit une bijection de $\mathfrak{n}$ sur $N$. On a montré plus haut que, si $n \in \mathfrak{n}$ et si $n \neq 0$, il existe un élément $h \in \mathfrak{h}_{z}+\mathfrak{w}$ tel que $[h, n] \neq 0$; dans ce cas, $\operatorname{ad}(\exp t h) n \neq n$ pour un nombre réel $t \neq 0$. Il en résulte que, pour tout élément $(\neq e)$ de $N$, il existe un élément de $H_{z} W$ qui ne commutent pas avec cet élément de $N$. En particulier, on a $N \cap\left(B^{c} W\right)=(e)$. Compte tenu de (2.7), on a donc:

$$
U=\left(B^{c} W\right) N, \quad\left(B^{c} W\right) \cap N=(e) .
$$

Par ailleurs, $(2,8)$ implique que

$$
U^{\prime} \supset N
$$

Le sous-groupe $W$ est simplement connexe et on $a$ :

$$
B^{c} \cap W=(e) .
$$

En effet, la restriction à $\mathfrak{h}$ de exp: $\mathfrak{g} \rightarrow G$ étant un homomorphisme du groupe abelien $\mathfrak{h}$ sur $H$ dont le noyau est dans $\mathfrak{h}_{k}=\mathfrak{h} \cap \mathfrak{f}$, exp induit un homomorphisme bijectif de $\mathfrak{w}$ sur $W$; il en résulte que $W$ est simplement connexe et que 
$\left(H_{s} H_{z}\right) \cap W=(e)$. Puisque le groupe $B^{c}$ est réductif, le centre de $B^{c}$ est contenu dans le sous-groupe de Cartan $H_{s} H_{z}$ de $B^{c}$. Puisque $\left[\mathfrak{b}^{c}, \mathfrak{w}\right]=0, B^{c} \cap W$ est dans le centre de $B^{c}$. Par conséquent, $B^{c} \cap W=H_{s} H_{z} \cap W=(e)$.

On considère maintenant le groupe $B^{c}$. Il résulte de $(2,6)$ que

$$
B^{c}=B_{s}^{c} H_{z} .
$$

et que $B_{s}^{c} \cap H_{z}$ est un sous-groupe discret de $B_{s}^{c}$ contenu dans le centre de $B_{s}^{c}$. L'algèbre de Lie complexe semi-simple $b_{s}^{c}$ admet comme forme réelle l'algèbre de Lie $\mathfrak{b}_{s}=\mathfrak{b}_{s}^{c} \cap \mathfrak{f}$ qui correspond au sous-groupe $B_{s}$ des commutateurs de $B$. Par conséquent, le groupe $B_{s}$ est un sous-groupe compact maximal du groupe semi-simple $B_{s}^{c}$ et contient donc le centre de $B_{s}^{c}$. D'autre part, l'algèbre de Lie abelienne $\mathfrak{h}_{z} \cap \mathfrak{t}$ est une forme réelle de $\mathfrak{h}_{z}$ et correspond à la composante connexe de l'élément neutre $C(B)$ du centre de $B$. Puisque la restriction à h de exp: $\mathfrak{g} \rightarrow G$ est un homomorphisme du groupe abelien $\mathfrak{h}$ sur $H$ dont le noyau est contenu dans $\mathfrak{h}_{k}=\mathfrak{h} \cap \mathfrak{f}$, il en résulte que $C(B)$ est le sous-groupe compact maximal du groupe abelien $H_{z}$. On a ainsi :

$$
B_{s}^{c} \cap H_{z}=B_{s} \cap H_{z}=B_{s} \cap C(B) .
$$

Cela fait, on va considérer les groupes $\operatorname{Hom}_{h}(U, A), \operatorname{Hom}_{h}\left(U, C^{m}\right)$ et Hom $(B, A)$, où le dernier groupe est le groupe des homomorphismes différentiables de $B$ dans $A$. La projection holomorphe $\pi: C^{m} \rightarrow A$ et l'injection $B \rightarrow U$ définissent de manière evidente les homomorphismes $\pi^{\prime}: \operatorname{Horin}_{h}\left(U, C^{m}\right)$ $\rightarrow \operatorname{Hom}_{h}(U, A)$ et $\lambda: \operatorname{Hom}_{h}(U, A) \rightarrow \operatorname{Hom}(B, A)$ respectivement,

Lemme 2. La suite

$$
(0) \longrightarrow \operatorname{Hom}_{h}\left(U, C^{m}\right) \stackrel{\pi^{\prime}}{\longrightarrow} \operatorname{Hom}_{h}(U, A) \stackrel{\lambda}{\longrightarrow} \operatorname{Hom}(B, A) \longrightarrow(0)
$$

est exacte.

Démonstration. Soit $f \in \operatorname{Hom}(B, A)$; la restriction de $f$ au sous-groupe $C(B)$ est un homomorphisme différentiable de $C(B)$ dans $A$ qui s'annule dans $B_{s} \cap C(B)$. Puisque l'algèbre de Lie $\mathfrak{h}_{z} \cap \mathfrak{t}$ de $C(B)$ est une forme réelle de $\mathfrak{h}_{z}$ et que $C(B)$ est le sous-groupe compact maximal du groupe abelien complexe $H_{z}, f$ se prolonge en un homomorphisme holomorphe bien déterminé $f^{c}: H_{z} \rightarrow A$. Il résulte de $(2,12)$ et de $(2,13)$ que $f^{c}$ se prolonge alors en un homomorphisme holomorphe $F: B^{c} \rightarrow A ; F$ est uniquement déterminé par $f^{c}$ et, par conséquent, 
par $f$, car $B_{s}^{c}$ est le sous-groupe des commutateurs de $B^{c}$. Les éléments de $W$ commütant avec les éléments de $B^{c}$, il résulte de $(2,11)$ que le groupe $B^{c} W$ est produit direct de $B^{c}$ et de $W$. L'homomorphisme $F$ se prolonge donc en un homomorphisme holomorphe $F: B^{c} W \rightarrow A$. D'après $(2,9)$, la restriction au sousgroupe $B^{c} W$ de l'homomorphisme canonique $U \rightarrow U / N$ est un isomorphisme de $B^{c} W$ sur $U / N$. Il en résulte que $F: B^{c} W \rightarrow A$ se prolonge en un homomorphisme holomorphe de $U$ dans $A$. Cela démontre que $\lambda$ est surjectif.

Puisque $B$ est un groupe compact, la restriction à $B$ d'un homomorphisme de $U$ dans $C^{m}$ est triviale. Il en résulte immédiatemaent que l'image de $\pi^{\prime}$ est contenue dans le noyau de $\lambda$.

Soit $F \in \operatorname{Hom}_{h}(U, A)$; le groupe $A$ étant abelien, $F$ induit l'homomorphisme trivial dans le sous-groupe $U^{\prime}$ des commutateurs de $U$. D'aprés $(2,10)$, la restriction de $F$ au sous-groupe $N$ est donc l'homomorphisme trivial. D'autre part, d'aprés la première partie de cette démonstration, la restriction de $F$ au sousgroupe $B$ détermine les valeurs de $F$ dans $B^{c}$. Supposons que $\lambda(F)=0$; la restriction de $F$ à $B$ est l'homomorphisme trivial et il en est donc le même pour la restriction de $F$ à $B^{c}$. D'après $(2,9)$ et $(2.11)$, on a la projection holomorphe $p_{W}: U \rightarrow W$ dont le noyau est $B^{c} N$. Il existe alors un homomorphisme holomorphe $F^{\prime}: W \rightarrow A$ tel que $F=F^{\prime} p_{W}$. Puisque $W$ est simplement connexe, il existe un homomorphisme holomorphe $\widetilde{F}^{\prime}: W \rightarrow C^{m}$ tel que $\pi \widetilde{F}^{\prime}=F^{\prime}$. L'homomorphisme holomorphe $\widetilde{F}: U \rightarrow C^{m}$ étant défini par $\widetilde{F}=\widetilde{F}^{\prime} p_{w}$, on a $\pi \widetilde{F}=F$, c'est-à-dire, $\pi^{\prime}(\widetilde{F})=F$. Cela démontre que le noyau de $\lambda$ est contenu dans l'image de $\pi^{\prime}$. Le Lemme 2 est ainsi démontré.

3. Démonstration des Théorèmes 2 et 3. Conservant les notations des sections précédentes, on considère le diagramme suivant.
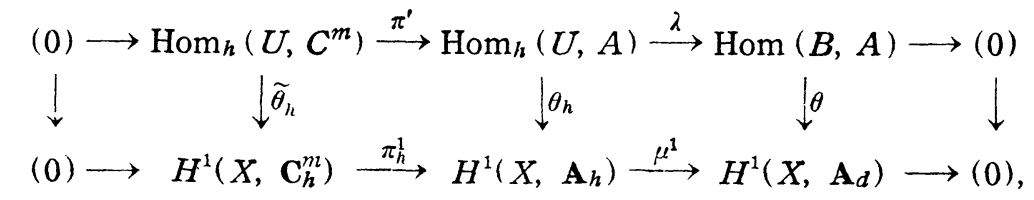

où $\theta, \theta_{h}, \widetilde{\theta}_{h}$ sont les homomorphismes définis au $\S 1.1$ et au $\S 2.1$. La première ligne est une suite exacte (Lemme 2).

On montre que ce diagramme est commutatif. Soient $F \in \operatorname{Hom}_{h}(U, A)$ et $f=\lambda(F) \in \operatorname{Hom}(B, A)$. D'après $\S 1.2, \theta(f)$ est la classe de l'espace fibré $P_{f}$ associé à l'espace fibré $K$ de base $X=K / B$ et de groupe $B$ par $f: B \rightarrow A$, où 
$P_{f}$ est l'espace quotient du produit $K \times A$ par la relation d'équivalence $(k, a)$ $\sim\left(k s^{-1}, f(s) a\right)$ quel que soit $s \in B$. De même, $\theta_{h}(F)$ est la classe de l'espace fibré $P_{F}$ associé à l'espace fibré $G$ de base $X=G / U$ et de groupe $U$ par $F: U \rightarrow A$, où $P_{F}$ est l'espace quotient du produit $G \times A$ par la relation d'équivalence $(y, a)$ $\sim\left(y s^{-1}, F(s) a\right)$ quel que soit $s \in U$. Or, puisque $G$ et $K$ sont transitifs sur $X$, on a $G=K U$. Il en résulte que l'injection différentiable de $K \times A$ dans $G \times A$ définit une bijection différentiable de $P_{f}$ sur $P_{F}$ qui commute avec les opérations de $A ; P_{f}$ et $P_{F}$ sont ainsi différentiablement équivalents, ce qui démontre que $\theta(f)=\mu^{1} \theta_{h}(F)$. On a donc $\theta \lambda=\mu^{1} \theta_{h}$. Il est aussi facile de voir que $\theta_{h} \pi^{\prime}=\pi_{h}^{1} \widetilde{\theta}_{h}$.

D'après le Lemme $2, \lambda$ est surjectif et, d'après le Corollaire $2 \mathrm{du}$ Théorème $1, \theta$ est bijectif. Il résulte donc du diagramme commutatif $(2,14)$ que $\mu^{1}$ est surjectif. Compte tenu du Lemme 1 , on voit que la seconde ligne de $(2,14)$ est une suite exacte. Cela démontre le Théorème 2.

Quant au Théorème 3 , ce théorème sera établi lorsqu'il est démontré dans le cas où $A$ est le groupe additif $C$ des nombre complexes. En effet, s'il en est ainsi, le théorème est aussi vrai dans le cas où $A=C^{m}$, puisque le groupe $C^{m}$ est somme directe de $m$ groupes isomorphes à $C$. L'homomorphisme $\widetilde{\theta}_{h}$ dans le diagramme $(2,14)$ est donc bijectif. On sait déjà que les deux lignes de $(2,14)$ sont des suites exactes (Lemme 2 et Théorème 2 ) et que $\theta$ est bijectif (Corollaire $2 \mathrm{du}$ Théorème 1). Appliquant le lemme de cinq à $(2,14)$, on en conclut que $\theta_{h}$ est bijectif, ce qui démontre le Théorème 3 dans le cas général.

On montre le Théorème 3 dans le cas où $A=C$. Remarquons que le groupe Aut $(P)$ des automorphismes d'un espace fibré principal holomorphe $P$ de base $X$ compacte, muni de la topologie compacts-ouverts, est un groupe de Lie complexe qui opère holomorphiquement dans $P[10]$. On a l'homomorphisme canonique p du groupe Aut $(P)$ dans le groupe Aut $(X)$ des homéomorphismes holomorphes de la base $X$ qui associe à un automorphisme de $P$ l'homéomorphisme de $X$ qu'il induit. Par ailleurs, si un groupe $G$ opère holomorphiquement dans $X$, on a un homomorphisme q de $G$ dans Aut $(X)$ en associant à un élément $y \in G$ l'homéomorphisme q(y) qu'il définit.

Lemme 3. Soit $G$ un groupe de Lie complexe connexe et soit $U$ un sousgroupe complexe fermé de $G$; on considère $G$ comme espace fibré principal holomorphe de base $G / U$ et de groupe $U$ opérant par les translations à droite. Supposons que $G / U$ soit compacte. Alors, pour qu'un espace fibré principal holo- 
morphe $P$ de base $G / U$ et de groupe $A$ soit associé à l'espace fibre $G$ par un homo. morphisme holomorphe de $U$ dans $A$, il faut et il suffit qu'il existe un homomorphisme holomorphe $\mathbf{q}^{*}$ du groupe $G$ dans le groupe Aut $(P)$ tel que $\mathbf{p q}^{*}=\mathbf{q}$.

Démonstration. Soit $P$ un espace fibré principal holomorphe de base $G / U$ et de groupe $A$ associé à $G$ par un homomorphisme $F: U \rightarrow A$. Comme on a remarqué dans le $\S 1.2$, il existe alors une application holomorphe $q^{*}: G \rightarrow P$, compatible avec la projection $q: G \rightarrow G / U$, tell que

$$
q^{*}(y s)=q^{*}(y) F(s)
$$

quels que soient $y \in G, s \in U$. Puisque $p q^{*}=p$, tout élément de $P$ s'écrit sous la forme $q^{*}(y) a$ avec $y \in G$ et $a \in A$. Pour un élément $y^{\prime} \in G$, on définit une application $\mathbf{q}^{*}\left(y^{\prime}\right): P \rightarrow P$ en posant $\mathbf{q}^{*}\left(y^{\prime}\right)\left(q^{*}(y) a\right)=q^{*}\left(y^{\prime} y\right) a ; \mathbf{q}^{*}\left(y^{\prime}\right)$ est bien définie et est un automorphisme de l'espace fibré $P$. On constate que l'application $\mathbf{q}^{*}: G \rightarrow$ Aut $(P)$ ainsi obtenue est un homomorphisme holomorphe tel que $\mathbf{p q}^{*}=\mathbf{q}$.

Réciproquement, soit $P$ un espace fibré principal holomorphe de base $G / U$ et de groupe $A$ pour lequel il existe un homomorphisme holomorphe $\mathbf{q}^{*}$ de $G$ dans $\operatorname{Aut}(P)$ tel que $\mathbf{p q}^{*}=\mathbf{q}$. On choisit un point $x_{0}^{*} \in P$ dont l'image par la projection $p: P \rightarrow X$ est le point $x_{0}=q(e)$, et on définit une application $q^{*}: G \rightarrow P$ en posant:

$$
q^{*}(y)=\mathbf{q}^{*}(y) x_{0}^{*} \quad \text { pour } \quad y \in G
$$

L'application $q^{*}$ est une application holomorphe qui est compatible avec $q$ : $G$ $\rightarrow G / U$. Il en résulte facilement que l'image réciproque de $P$ par $q$ est un espace fibré trivial, c'est-à-dire que $P$ est trivialisé par $q$. Or, si $s \in U$, $p\left(\mathbf{q}^{*}(s) x_{0}^{*}\right)=\mathbf{p}(s) x_{0}=x_{0}=p\left(x_{0}^{*}\right)$ et il existe donc un élément $F(s) \in A$ tel que $\mathbf{q}^{*}(s) x_{0}^{*}=x_{0}^{*} F(s)$. Puisque $\mathbf{q}^{*}: G \rightarrow$ Aut $(P)$ est un homomorphisme, on a $q^{*}(y s)=\mathbf{q}^{*}(y s) x_{0}^{*}=\mathbf{q}^{*}(y) x_{0}^{*} F(s)=q^{*}(y) F(s)$, ce qui montre que l'application $F: U \rightarrow A$ est un facteur associé à $P$. L'application $F$ est donc un homomorphisme holomorphe de $U$ dans $A$ et l'espace fibré $P$ est un espace fibré associé à $G \operatorname{par} F$.

Démonstration du Théorème 3 dans le cas où $A=C$. Il faut montrer que l'homomorphisme $\theta_{h}: \operatorname{Hom}_{h}(U, C) \rightarrow H^{1}\left(X, \mathbf{C}_{h}\right)$ est bijectif.

1) $\theta_{h}$ est surjectif. Soit $P$ un espace fibré principal holomorphe de base 
$X$ et de groupe $A$. D'aprés un résultat de Bott [3], si $K$ est un groupe de Lie compact connexe opérant sur une $C$-variété $X$ de manière transitive par des transformations holomorphes, les opérations de $K$ induisent l'automorphisme identique du groupe $H^{1}\left(X, \mathbf{C}_{h}\right)$. Il en résulte que l'image réciproque de $P$ par une transformation $k: X \rightarrow X$ appartenant à $K$ est isomorphe sur $X$ à $P$; autrement dit, il existe un automorphisme de $P$ qui induit la transformation $k$ de la base $X$.

Supposons maintenant que $X=G / U=K / B$ au sens de $(2,2)$. Puisque $G$ est presque effectif sur $X$, l'homomorphisme $\mathbf{q}: G \rightarrow$ Aut $(X)$ est localement injectif. Il en résulte que l'image $\mathbf{q}(G)$ est le plus petit sous-groupe de Lie complexe connexe de Aut $(X)$ contenant $\mathbf{q}(K)$. Or, l'image $\mathbf{p}(\operatorname{Aut}(P))$ est un sousgroupe de Lie complexe de Aut $(X)$ et, d'après ce qui précède, elle contient le sous-groupe $\mathbf{q}(K)$. Par conséquent, $\mathbf{q}(G)$ est contenu dans le plus grand sousgroupe connexe de $\mathbf{p}(\operatorname{Aut}(P))$. Puisque $\mathbf{p}:$ Aut $(P) \rightarrow \mathbf{p}(\operatorname{Aut}(P))$ est une application ouverte, il en résulte que $\mathbf{q}(G) \subset \mathbf{p}\left(\operatorname{Aut}(P)^{\circ}\right)$ où Aut $(P)^{\circ}$ est le plus grand sous-groupe connexe de $\operatorname{Aut}(P)$. Il existe donc un sous-groupe de Lie complexe connexe $G^{*}$ de Aut $(P)^{\circ}$ tel que $\mathbf{p}\left(G^{*}\right)=\mathbf{q}(G)$. Le groupe $\mathbf{q}(G)$ étant un groupe de Lie complexe semi-simple, il existe alors un homomorphisme holomorphe local $\sigma$ d'un voisinage de l'élément neutre de $\mathbf{q}(G)$ dans $G^{*}$ tel que po soit identité. Puisque $G$ est simplement connexe, l'homomorphisme local oq se prolonge en un homomorphisme holomorphe $\mathbf{q}^{*}: G \rightarrow G^{*}$. On a ainsi un homomorphisme holomorphe $\mathbf{q}^{*}: G \rightarrow$ Aut $(P)$ tel que $\mathbf{p q}^{*}=\mathbf{q}$ et, d'après le Lemme 3 , on en conclut que $P$ est un espace fibré associé à l'espace fibré $G$ de base $X$ par un homomorphisme de $U$ dans $A$. Cela démontre que $\theta_{h}$ est surjectif.

2) $\theta_{h}$ est injectif. Soit $F \in \operatorname{Hom}_{h}(U, C)$ et supposons que $\theta_{h}(F)=0$; un espace fibré associé à $G$ par $F$ est trivial. D'après ce qu'on a vu au $\S 1.2$, il existe alors une fonction holomorphe $r$ sur $G$ à valeurs dans $C$ telle que

$$
F(s)=r(y)-r(y s)
$$

quels que soient $y \in G, s \in U$. Prenons la fonction $r_{1}$ sur $G$ définie par $r_{1}(y)$ $=\int_{K} r(k y) d k$ pour $y \in G$, où l'intégration est prise par rapport à la mesure invariante sur $K$ de volume total $1 ; r_{1}$ est une fonction holomorphe sur $G$, puisque les translations à gauche de $G$ par $K$ sont holomorphes. On a :

$$
F(s)=r_{1}(y)-r_{1}(y s)
$$


quels que soient $y \in G, s \in U$. Il en résulte en particulier que la forme $d r_{1}$ est une forme holomorphe sur $G$ qui est invariante par les translations à droite de $G$ par les éléments de $U$. D'autre part, puisque $r_{1}$ est constante dans $K$, la forme $d r_{1}$ induit la forme nulle dans la sous-variété réelle $K$ de $G$. L'espace tangent à $G$ en un point de $K$ est un espace vectoriel complexe engendré par le sous-espace vectoriel réel des vecteurs tangents à la sous-variété $K$, car $G$ est un groupe de Lie complexe semi-simple ayant $K$ comme sous-groupe compact maximal. Puisque $d r_{1}$ est une forme holomorphe, la forme $d r_{1}$ est donc nulle en tout point de $K$. Le groupe $K$ étant transitif sur $X=G / U$, on a $G=K U$. Puisque $d r_{1}$ est invariante par les translations à droite de $G$ par les éléments de $U$, il en résulte que la forme $d r_{1}$ est partout nulle sur $G$. Comme $G$ est connexe, cela signifie que $r_{1}$ est une fonction constante. D'après $(2,15)$, on a donc $F=0$, ce qui démontre que $\theta_{h}$ est injectif.

Le Théorème 3 est ainsi démontré dans le cas où $A=C$ et donc, comme on l'a vu plus haut, aussi dans le cas général.

§3. Connexions d'un espace fibré principal holomorphe de groupe abelien ayant pour base une $C$-variété

1. Connexions de type spécial.

Lemme 4. Soit $X$ une C-variété kaehlérienne et soit $K$ un groupe de Lie compact connexe semi-simple opérant sur $X$ par des transformations holomorphes de manière transitive et presque effective. Soit $C^{m}$ un espace vectoriel complexe de dimension $m$. Alors, 1) toute forme de degré 1 sur $X$ à valeurs dans $C^{m}$ invariante par $K$ est nulle; 2) pour qu'une forme $\Omega$ fermée de degré 2 sur $X$ à valeurs dans $C^{m}$ soit invariante par $K$ il faut et il suffit que $\Omega$ soit une forme harmonique par rapport à une métrique kaehlérienne sur $X$ invariante par $K$.

Démonstration. On peut supposer que $m=1$. Soit $B$ le sous-groupe de stabilité de $K$ en un point de $X ; X$ peut être identifiée avec l'espace homogène kaehlérien $K / B$. On sait que $B$ contient un sous-groupe de Cartan $H$ de $K$ [2]. Soient $\mathfrak{k}, \mathfrak{b}, \mathfrak{h}$ les algèbres de Lie de $K$, de $B$ et de $H$ respectivement. Comme $B$ est compact, il existe un sous-espace $m$ de $f$ tel que $f$ soit la somme directe $m+\mathfrak{b}$ et que $[\mathfrak{b}, m] \subset m$. Puisque $\mathfrak{h} \subset \mathfrak{b}$, on a alors $[\mathfrak{b}, m]=m$. Or, soit $\psi$ une forme de degré 1 sur $K / B$ invariante par $K$. L'image réciproque $\psi^{*}$ de $\psi$ par la projection de $K$ sur $K / B$ est une forme invariante ì gauche sur $K$ qui s'annule 
pour les vecteurs appartenant à $\mathfrak{b}$ et à $[\mathfrak{b}, \mathfrak{t}]$. Puisque $\mathfrak{f}=[\mathfrak{b}, \mathfrak{m}]+\mathfrak{b}$, on a alors $\psi^{*}=0$. Par suite $\psi=0$, ce qui démontre 1 ). Puisque $K$ est un groupe connexe opérant sur $X$, une forme sur $X$ qui est harmonique par rapport à une métrique kaehléreinne invariante par $K$ est invariante par $K$. Réciproquement, soit $\Omega$ une forme fermée de degré 2 sur $X$ invariante par $K$. On sait qu'il existe une forme harmonique $\Omega_{h}$ de degré 2 sur $X$ telle que $\Omega-\Omega_{h}$ soit une forme exacte [6]. Puisque $\Omega-\Omega_{h}$ est invariante par $K$ il existe alors une forme $\psi$ de degré 1 sur $X$ invariante par $K$ telle que $\Omega-\Omega_{h}=d \psi$ [5]. D'après 1 ), on a $\psi=0$ et par suite $\Omega=\Omega_{h}$, ce qui démontre que $\Omega$ est une forme harmonique.

ThÉ́nème 4. Soit $X$ une $C$-variété et soit $K$ un groupe de Lie compact connexe semi-simple qui opère sur $X$ par des transformations holomorphes de manière transitive et presque effective. Un espace fibré principal holomorphe $P$ de base $X$ et de groupe $A$ admet une connexion de type $(1,0)$ telle que sa forme de courbure sur $X$ soit une forme de type $(1,1)$ invariante par les opérations de $K$ sur $X$. Si $X$ est kaehlérienne, cette connexion est unique.

Démonstration. On montre d'abord que l'on peut supposer que $K$ est le groupe construit au $\S 2.2$. On sait que le revêtement universel du groupe compact semi-simple $K$ est un revêtement fini de $K$ qui est aussi compact. En remplaçant au besoin $K$ par ce dernier groupe, on peut donc'supposer que le groupe $K$ est simplement connexe. D'après l'hypothèse, on a un homomorphisme q de $K$ dans le groupe Aut $(X)$ des homéomorphismes holomorphes de $X$ dont le noyau est un sous-groupe discret de $K$. Par conséquent, l'homomorphisme de l'algèbre de Lie $\ddagger$ de $K$ dans l'algèbre de Lie $\mathfrak{f}$ de Aut $(X)$ induit par q est injectif. Désignons encore par $\mathbf{q}$ cet homomorphisme d'algebres de Lie. Puisque

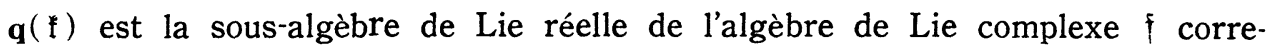

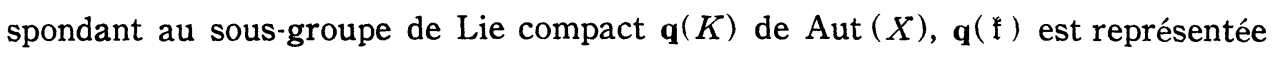
par des matrices anti-hermitiennes lorsque l'on choisit une représentation fidéle de l'algèbre de Lie complexe $f$ de manière convenable. Il en résulte que les

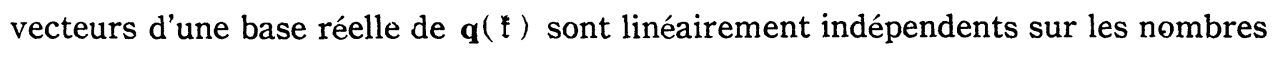
complexes dans $f$. Par conséquent, $g$ étant la complexification de $\mathfrak{k}$, l'homomorphisme injectif $\mathbf{q}: \mathfrak{f} \rightarrow \mathfrak{f}$ se prolonge en un homomorphisme injectif $\mathbf{q}$ de l'algèbre de Lie complexe $g$ dans $\mathfrak{f}$. Soit $G$ le groupe de Lie complexe simplement connexe ayant $g$ pour algèbre de Lie. Puisque $K$ est un groupe de Lie 
compact simplement connexe dont l'algèbre de Lie est la forme réelle $\mathfrak{t}$ de $\mathfrak{g}$, on peut considérer que $G$ contient $K$ comme le sous-groupe compact maximal correspondant à la sous-algèbre de Lie t. Puisque $G$ est simplement connexe, il existe un homomorphisme holomorphe, désigné encore par $\mathbf{q}$, du groupe $G$ dans le groupe Aut $(X)$ qui induit l'homomorphisme $\mathbf{q}: \mathfrak{g} \rightarrow \mathfrak{f}$. Il est alors clair que la restriction de $\mathbf{q}: G \rightarrow \operatorname{Aut}(X)$ à $K$ coïncide avec $\mathbf{q}: K \rightarrow \operatorname{Aut}(X)$. Il en résulte que le groupe $G$ opère sur $X$ holomorphiquement et de manière transitive. Puisque l'homomorphisme $\mathbf{q}: \mathfrak{g} \rightarrow \mathfrak{f}$ induit par $\mathbf{q}: G \rightarrow$ Aut $(X)$ est injectif, le noyau de ce dernier homomorphisme est un sous-groupe $\operatorname{discret} \operatorname{de} G$; le groupe $G$ opère donc sur $X$ de manière presque effective.

On se trouve ainsi à la situation considérée au début du $\$ 2.2$. Or, on y a défini une décomposition de Cartan par rapport à laquelle l'algèbre de Lie $\mathfrak{u}_{1}$ du sous-groupe de stabilité $U_{1}$ en un point $x_{1}$ s'écrit sous la forme $(2,4)$. Soit $K_{1}$ le sous-groupe compact maximal de $G$ correspondant à la forme compacte $(2,3)$ par rapport à cette décomposition de Cartan. On sait que deux sous-groupes compacts maximaux de $G$ sont conjugués; il existe donc un élément $y \in G$ tel que $y K_{1} y^{-1}=K$. Prenons la transformée par ad $(y)$ de la décomposition de Cartan donnée plus haut. Il est evident que l'algèbre de Lie $\mathfrak{l}^{\prime}$ de $K$ et l'algèbre de Lie $\mathfrak{u}$ du groupe $U=y U_{1} y^{-1}$ sont alors représentées, par rapport à cette nouvelle décomposition de Cartan, par les formes $(2,3)$ et $(2,4)$ respectivement. Puisque $U$ est le sous-groupe de stabilité de $G$ en le point $x_{0}=y x_{1}$, cela montre que le groupe $K$ est le groupe construit par le procédé du $\S 2.2$ à partir d'un groupe de Lie $G$ opérant sur $X$ et du sous-groupe de stabilité $U$ de $G$ en un point $x_{0} \in X$.

Cela dit, considérons l'espace fibré principal holomorphe $G$ de base $X$ et de groupe $U$ dont la projection $q$ est définie par $q(y)=y x_{0}$ pour $y \in G$. La restriction de $q$ à $K \subset G$ est la projection du sous-espace fibré principal différentiable $K$ de groupe $B$. De plus, le groupe $K$ étant transitif sur $X$, on a $G=K U$ et $K \cap U=B$; tout élément $y \in G$ est de la forme $k s$ avec $k \in K$ et $s \in U$, et $k s=k^{\prime} s^{\prime}$ si et seulement si il existe un élément $t \in B$ tel que $s^{\prime}=t s$. On va définir maintenant une connexion de l'espace fibré principal $G$. D'après $\$ 2.2$, g se décompose en somme directe de deux sous-espaces

$$
\mathfrak{g}=\mathfrak{p}+\mathfrak{u}, \quad \text { où } \quad \mathfrak{p}=\overline{\mathfrak{w}}+\sum_{\alpha \in S_{+}}\left\{\boldsymbol{e}_{\alpha}\right\}^{c} .
$$

Si $s \in U$, alors ad $\left(s^{-1}\right) \mathfrak{u}=\mathfrak{u}$, donc 


$$
g=\operatorname{ad}\left(s^{-1}\right) \mathfrak{p}+\mathfrak{n}
$$

est aussi une décomposition en somme directe de $g$. On observe que si $t \in B$ alors $\operatorname{ad}\left(t^{-1}\right) \mathfrak{p}=\mathfrak{p}$, puisque $\left[\mathfrak{b}^{c}, \mathfrak{p}\right] \subset \mathfrak{p}$ d'après $(2,5)$. Posons

$$
c(y)=y\left(\operatorname{ad}\left(s^{-1}\right) \mathfrak{p}\right)
$$

pour $y=k s$ avec $k \in K$ et $s \in U ; c(y)$ est le sous-espace de l'espace tangent à $G$ en le point $y$ formés par les transformées des vecteurs appartenant à $\operatorname{ad}\left(s^{-1}\right) \mathfrak{p}$ par la translation à gauche de $G$ par l'élément $y$. D'après ce qu'on vient de voir, $c(y)$ est uniquement déterminé par $y \in G$. De plus, on constate immédiatement que le champ de sous-espaces tangents $\{c(y) ; y \in G\}$ est une connexion de l'espace fibré principal $G$. Soit $r$ la forme de cette connexion. Puisque le tenseur de la structure complexe de $G$ est invariant à gauche et induit la mutiplication par $i$ dans $g$, ce tenseur applique le champ $\{c(y) ; y \in G\}$ en lui-même, ce qui signifie que la forme $\gamma$ est de type $(1,0)$ sur $G$.

Soit maintenant $P$ un espace fibré principal holomorphe de base $X$ et de groupe $A$. D'après le Théorème $3, P$ est un espace fibré associé à $G$ par un homomorphisme $F: U \rightarrow A$ et on a donc la connexion de $P$ canoniquement associée à la connexion $\gamma$ de $G$ définie plus haut (cf. \$1.2). On démontrera que cette connexion vérifie les conditions du théorème. Soit $\omega$ la forme de cette connexion. Puisque $r$ est une forme de type $(1,0)$ sur $G$, on voit facilement que $\omega$ est une forme de type $(1,0)$ sur $P$, c'est-à-dire que la connexion est de type $(1,0)$. On montre que la forme de courbure $\Omega$ de cette connexion est une forme de type $(1,1)$ invariante par $K$ sur $X$. Soit $\gamma_{0}$ la forme invariante à gauche sur $G$ à valeurs dans $\mathfrak{u}$ qui est définie par la projection de $\mathfrak{g}$ sur $\mathfrak{u}$ de noyan $\mathfrak{p}$. Puisque $\mathrm{c}(k)=k \mathfrak{p}$ pour $k \in K$, la restriction de $\gamma_{0}$ à la sous-variété réelle $K$ coïncide avec la restriction de la forme de connexion $\gamma$ à $K$. Par conséquent, $\mid K$ signifiant la restriction des formes sur $G$ à $K$, on a $\gamma\left|K=\gamma_{0}\right| K$ et par suite

$$
(d \gamma)\left|K=\left(d \gamma_{0}\right)\right| K
$$

D'après $(1,5)$, on a donc

$$
(\Omega q) \mid K=F\left(\left(d r_{0}\right) \mid K\right) .
$$

Puisque la forme $F\left(\left(d r_{0}\right) \mid K\right)$ est une forme invariante à gauche sur $K$, il en résulte que la forme $\Omega$ est une forme invariante par les opérations de $K$ sur $X$, 
Pour voir que la forme $\Omega$ est de type $(1,1)$ il suffit alors de le verifier au point $x_{0} \in X$, puisque $K$ opère transitivement sur $X$ par les transformations holomorphes. La projection $q$ de $G$ sur $X$ induit une application linéaire de $\mathfrak{g}$ sur l'espace tangent $X_{0}$ au point $x_{0}$ de $X$. Le noyau de cette application étant $\mathfrak{u}$, la restricition de cette application à $p$ est bijective; de plus la multiplication par $i$ dans $p$ correspond dans $X_{0}$ au tenseur de la structure complexe de $X$. Par conséquent, $\Omega$ est de type $(1,1)$ si $(\Omega q)(i x, i y)=(\Omega q)(x, y)$ quels que soient $x, y \in \mathfrak{p}$. D'après $(1,5)$ cette condition signifie que

$$
F(\Gamma(i x, i y))=F(\Gamma(x, y))
$$

quels que soient $x, y \in \mathfrak{p}$, où $\Gamma$ est la forme de courbure sur $G$ de la connexion définie par $\gamma$. Or, pour vérifier $(3,3)$, supposons donné pour chaque élément $x \in \mathfrak{p}$ un élément $x_{k} \in \mathfrak{f}$ tel que $x_{k} \equiv x(\bmod \mathfrak{u})$. Si $x, y \in \mathfrak{p}$, on a alors

$$
\Gamma(x, y)=\Gamma\left(x_{k}, y_{k}\right)
$$

d'après la definition de la forme $\Gamma$ et, compte tenu de $(1,5)$ et de $(3,1)$, on a donc $F(\Gamma(x, y))=F\left(\Gamma\left(x_{k}, y_{k}\right)\right)=F\left(d r\left(x_{k}, y_{k}\right)\right)=F\left(d r_{0}\left(x_{k}, y_{k}\right)\right)=F\left(\gamma_{0}\left(\left[x_{k}, y_{k}\right]\right)\right)$, la dernière égalité résultant $d u$ fait que $\gamma_{0}$ est une forme invariante à gauche sur $G$. Par conséquent, la formule $(3,3)$ est équivalente à :

$$
F\left(\gamma_{0}\left(\left[(i x)_{k},(i y)_{k}\right]\right)\right)=F\left(\gamma_{0}\left(\left[x_{k}, y_{k}\right]\right)\right) .
$$

Cela dit, on montre $(3,3)$. L'espace $p$ est engendré sur les nombres réels par les vecteurs $h \in \overline{\mathfrak{w}}, e_{\alpha}$ et $i e_{\alpha}$ avec $\alpha \in S_{+}$. Puisque les deux membres de $(3,3)$ sont bilinéaires sur les nombres réels, il suffit de vérifier $(3,3)$ dans le cas où $x$ et $y$ sont de la forme $h, e_{\alpha}$ ou $i e_{\alpha}$ avec $\alpha \in S_{+}$. On peut poser $h_{k}=h+\bar{h}$ pour $h \in \overline{\mathfrak{w}}$ et $\left(e_{\alpha}\right)_{k}=u_{\alpha},\left(i e_{\alpha}\right)_{k}=v_{\alpha}$ pour $\alpha \in S_{+}$, où $u_{\alpha}, v_{\alpha}$ sont les vecteurs définis dans $(2,3)$. Alors, sauf dans le cas où $x=e_{\alpha}$ et $y=i e_{\alpha}$, on constate que $r_{0}\left(\left[(i x)_{k},(i y)_{k}\right]\right)$ et $r_{0}\left(\left[x_{k}, y_{k}\right]\right)$ sont dans $\sum_{\alpha \in s_{0} \cup S_{-}}\left\{e_{\alpha}\right\}^{c}$ qui est contenu dans $\mathfrak{u}^{\prime}$ d'après $(2,8)$ et que les deux membres de $(3,5)$ sont donc nuls. Si $x=e_{\alpha}$ et $y=i e_{\alpha}$, alors $x_{k}=u_{\alpha}, y_{k}=v_{\alpha},(i x)_{k}=v_{\alpha},(i y)_{k}=-u_{\alpha}$ et $(3,5)$ est trivialement vérifiée. D'après la remarque ci-dessus, cela démontré $(3,3)$; la forme $\Omega$ est de type $(1,1)$ sur $X$.

Montrons la dernière partie du théorème où l'on suppose que $X$ est kaehlérienne. Soient $\omega$ et $\omega^{\prime}$ deux formes de connexion de type $(1,0)$ dont les formes de courbure $\Omega$ et $\Omega^{\prime}$ sont de type $(1,1)$ et invariantes par $K$. Puisque 
le groupe $A$ est abelien, il existe alors une forme $\xi$ de type $(1,0)$ sur $X$, à valeurs dans $\mathfrak{a}$, telle que $\omega-\omega^{\prime}=\xi p$. Il en résulte pue $\Omega-\Omega^{\prime}=d \xi$. Puisque $\Omega$ et $\Omega^{\prime}$ sont harmoniques par rapport à une métrique kaehlérienne sur $X$ (Lemme 4), cela implique $\Omega=\Omega^{\prime}$ et par suite $d \xi=0$. La forme $\xi$ est donc holomorphe et par conséquent harmonique. Puisque le premier nombre de Betti de $X$ est nulle, on a $\xi=0$, ce qui démontre que $\omega=\omega^{\prime}$. Le Théorème 4 est ainsi démontré.

2. Connexions holomorphes. Le but de cette section est d'établir le théorème suivant.

THÉonème 5. Soit $P$ un espace fibré principal de groupe $A$ ayant pour base une C-variété $X$. Si $P$ admet une connexion holomorphe, $P$ est un espace fibré trivial.

Pour démontrer ce théorème on emploie la fibration de Wang d'une $C$ variété $X$ par un groupe abelien, la base étant une $C$-variéte kaehlerienne. Soit $X=G / U=K / B$ au sens de $(2,2)$ où $K$ est le sous-groupe compact maximal de $G$ construit dans $\S 2.2$. On utilisera les notations du $\S 2.2$. Posons

$$
\hat{\mathfrak{u}}=\mathfrak{h}+\sum_{\alpha \in S_{0}}\left\{\boldsymbol{e}_{\alpha}\right\}^{c}+\sum_{\alpha \in S_{-}}\left\{\boldsymbol{e}_{\alpha}\right\}^{c}
$$

D'après Wang [12], $\hat{\mathfrak{u}}$ correspond à un sous-groupe de Lie complexe connexe fermé $\hat{U}$ dans $G$ et l'espace homogène complexe $\hat{X}=G / \hat{U}$ est une $C$-variété kaehlérienne. Il résulte de $(2,8)$ que la sous-algèbre des commutateurs de $\hat{\imath}$ coïncide avec la sous-algebre $\mathfrak{u}^{\prime}$ des commutateus de $\mathfrak{u}$; par conséquent, le quotient $T=\hat{U} / U$ est un groupe de Lie complexe abelien connexe. Les opérations de $T$ dans $X=G / U$ définissent une structure d'espace fibré principal holomorphe de base $\hat{X}=G / \hat{U}$ et de groupe $T$. On désignera par $\hat{p}$ la projection de cet espace fibré $X ; \hat{p}$ commute avec les opérations de $G$ dans $X$ et dans $\hat{X}$. Si $\hat{B}=\hat{U} \cap K$, on a donc $U \hat{B}=\hat{U}$. Par consèquent, les opérations du groupe $T=\hat{U} / U$ dans $X$ sont induites par les opérations du groupe $\hat{B}$ dans $X$.

D'après le Théorème 4, l'espace fibré principal $X$ de base $\hat{X}$ et de groupe $T$ admet une connexion bien déterminée dont la forme $\omega$ est de type $(1,0)$ et dont la forme de courbure $\Omega$ est une forme de type $(1,1)$ sur $X$ invariante par $K$. La forme $d \omega=\Omega \hat{p}$ est alors de type $(1,1)$; par conséquent, on a :

$$
d^{\prime \prime} \omega=d \omega .
$$

On observe de plus que $\omega$ est une forme sur $X$ invariante par $K$. En effet, 
chaque élément $k \in K$ définissant un automorphisme de l'espace fibré $X$, la transformée $\omega k$ de la forme $\omega$ est une forme de connexion de type $(1,0)$ dont la forme de courbure est égale à $\Omega k$. Puisque $\Omega$ est invariante par $K ; \Omega k=\Omega$, on a $\omega k=\omega$ d'après l'unicité de la connexion du type considéré.

Soit $\mathscr{E}^{(q)}(X)$ le module des formes différentielles de degré $q$ sur $X$ à valeurs complexes. La somme directe $\mathscr{E}(X)=\sum_{q \geqq 0} \mathscr{E}^{(q)}(X)$ est l'algèbre graduée des formes sur $X$. Les formes sur $X$ qui sont invariantes par les opérations de $K$ sur $X$ forment une sous-algèbre graduée $\mathscr{E}_{I}(X)=\sum_{q \geqq 0} \mathscr{E}_{I}^{(q)}(X)$ de $\mathscr{E}(X)$. On définit de même les algébres graduées $\mathscr{E}(\hat{X})=\sum_{q \geqq 0} \mathscr{E}^{(q)}(\stackrel{\hat{X}}{)})$ et $\mathscr{E}_{I}(\hat{X})=\sum_{q \geqq 0} \mathscr{E}_{I}^{(q)}(\hat{X})$. La projection $\hat{p}$ de $X$ sur $\hat{X}$ induit un homomorphisme injectif de $\mathscr{C}(\hat{X})$ dans $\mathscr{E}(X)$. Soit d'autre part $t$ l'algèbre de Lie du groupe $T ; t$ est une algèbre de Lie complexe abelienne. Désignons par $t^{c}$ la complexification de $t$ en prenant t comme algèbre de Lie réelle. La variété $X$ étant un espace fibré principal de groupe $T$, on a une anti-dérivation $i(z)$ de l'algèbre graduée $\mathscr{E}(X)$ pour chaque $z \in 1^{c}$ [4]; on observe que les transformations de $\mathscr{E}(X)$ induites par les opérations de $K$ sur $X$ commutent avec $i(z)$ quel que soit $z \in \mathrm{t}^{c}$, puisque les opérations de $K$ sur $X$ sont des automorphismes de l'espace fibré principal $X$. On sait que la forme $\phi \hat{p}$, image réciproque d'une forme $\psi \in \mathscr{E}(\hat{X})$ par $\hat{p}: X \rightarrow \hat{X}$, est annulée par $i(z)$ quel que soit $z \in t^{c}$.

Choisissons maintenant une base complexe $\left\{e_{1}, \ldots, e_{n}\right\}$ de l'algèbre de Lie complexe $\mathrm{t}(n=$ dimension complexe de $\mathrm{t})$, et prenons les éléments de $\mathrm{t}^{c}$ :

$$
\begin{aligned}
& z_{1}=e_{1}-\sqrt{-1} i e_{1}, \ldots, z_{n}=e_{n}-\sqrt{-1} i e_{n}, \\
& z_{n+1}=e_{1}+\sqrt{-1} i e_{1}, \ldots, z_{2 n}=e_{n}+\sqrt{-1} i e_{n},
\end{aligned}
$$

où $\sqrt{ }-1$ (resp. $i$ ) est la structure complexe de $t^{c}$ (resp. de $t$ ); on a ainsi une base complexe $\left\{z_{i}, \ldots, z_{2 n}\right\}$ de $t^{c}$. Soient $g^{1}, \ldots, g^{2 n}$ les fonctions linéaires complexes sur $t^{c}$ telles que $g^{j}\left(z_{l}\right)=\delta_{j l}$ pour $j, l=1, \ldots, 2 n$. On pose

$$
\omega^{j}=g^{j}(\omega) \quad \text { pour } \quad j=1, \ldots, 2 n \text {. }
$$

Les formes $\omega^{1}, \ldots, \omega^{n}$ (resp. $\omega^{n+1}, \ldots, \omega^{2 n}$ ) sont des formes de type $(1,0)$ (resp. de type $(0,1)$ ) invariantes par les opérations de $K$ sur $X$. On a de plus:

$$
i\left(z_{l}\right) \omega^{j}=\delta_{j l} \quad \text { pour } j, l=1, \ldots, 2 n .
$$

Par ailleurs, d'après (3.6), $d^{\prime \prime} g^{i}(\omega)=g^{i}\left(d^{\prime \prime} \omega\right)=g^{i}(d \omega)=d g^{i}(\omega)$ pour $i=1, \ldots$; $n$; on a donc: 


$$
d^{\prime \prime} \omega^{i}=d \omega^{i} \quad \text { pour } \quad i=1, \ldots, n .
$$

Lemme 5. Toute forme $\varphi \in \mathscr{E}^{(a)}(X)$ s'écrit d'une manière et d'une seule sous la forme

$$
\varphi=\sum_{r=0}^{q} \sum_{1 \leqq j_{1}<\cdots<j_{r} \leqq 2 n} \varphi_{j_{1} \cdots j_{r}}^{(q-r)} \wedge \omega^{j_{1}} \wedge \cdots \wedge \omega^{j_{r}}
$$

oì $\varphi_{j_{1} \cdots j r}^{(q-r)} \in \mathscr{C}^{(q-r)}(X)$ et où

$$
i(z) \varphi_{j_{1} \cdots j_{r}}^{(q-r)}=0
$$

quel que soit $z \in t^{c}$. De plus, pour que $\varphi \in \mathscr{C}_{1}(X)$, il faut et il suffit qu'il existe des formes $\psi_{j_{1} \cdots j_{r}}^{(q-r)} \in \mathscr{C}_{I}^{(q-r)}(\hat{X})$ telles que $\varphi_{j_{1} \cdots j_{r}}^{(q-r)}=\psi_{j_{1} \ldots j_{r}}^{(q-r)} \hat{p}\left(r=0, \ldots, q, 1 \leqq j_{1}\right.$ $\left.<\cdots<j_{r} \leqq 2 n\right)$.

- Démonstration. Les images réciproques $\phi \hat{p} \cdot$ des formes $\psi \in \mathscr{E}(\hat{X})$ par $\hat{p}: X \rightarrow \hat{X}$ et les formes $\omega^{1}, \ldots, \omega^{2 n}$ engendrent l'algèbre $\mathscr{E}(X)$ sur le module des fonctions différentiables sur $X$, car les restrictions de ces formes à chaque point de $X$ engendrent l'algèbre sur les nombres complexes consitituée par les formes multilinéaires antisymétriques sur l'espace tangent à $X$ en ce point. Puisque le produit de la forme $\psi \hat{p}$, image réciproque d'une forme $\psi \in \mathscr{C}(\hat{X})$ par $\hat{p}: X \rightarrow \hat{X}$, par une fonction sur $X$ est annulé par $i(z)$ quel que soit $z \in t^{c}$, il en résulte que toute forme $\varphi \in \mathscr{C}^{(q)}(X)$ est de la forme $(3,9)$. Pour voir l'unicité de l'expréssion (3.9) de $\varphi$, il suffit de montrer que, si $\varphi=0$, alors $\varphi_{j_{1} \cdots j_{r}}^{(q-r)}=0$ pour tout $r=0, \ldots, g$ et $1 \leqq j_{1}<\cdots<j_{r} \leqq 2 n$. Supposons que, si $r>r_{0}, \varphi_{j_{1} \ldots j_{r}}^{(q-r)}=0$ pour tout $1 \leqq j_{1}<\cdots<j_{r} \leqq 2 n$. Si $r_{0}>0$, il résulte de $(3,7)$ que $\varphi_{j_{1} \cdots j_{r_{0}}}^{\left(q-r_{0}\right)}=(-1)^{\left(q-r_{0}\right) r_{0}} i\left(z_{j_{r_{0}}}\right) \cdots i\left(z_{j_{1}}\right) \varphi=0$ pour tout $1 \leqq j_{1}<\cdots<j_{r_{0}} \leqq 2 n$. Si $r_{0}=0, \varphi^{(q)}=\varphi=0$ et l'unicité de $(3,9)$ est démontré.

Montrons la seconde partie du lemme. Étant donnée une forme $\varphi \in \mathscr{E}^{(q)}(X)$ écrite sous la forme $(3,9)$, sa transformée $\varphi k$ par l'opération d'un élément $k \in K$ est représentée par

$$
\varphi k=\sum_{r=0}^{q} \sum_{1 \leqq j_{1}<\cdots<j_{r} \leqq 2 n}\left(\varphi_{j_{1} \cdots j_{r}}^{(q-r)} k\right) \wedge \omega^{j_{1}} \wedge \cdots \wedge \omega^{j_{r}}
$$

puisque $k$ induit un automorphisme de l'algèbre $\mathscr{C}(X)$ et que $\omega^{1}, \ldots ; \omega^{2 n}$ sont des formes invariantes par $K$. On a remarqué plus haut que, si $\varphi_{*} \in \mathscr{E}(X)$, $i(z)\left(\varphi_{*} k\right)=\left(i(z) \varphi_{*}\right) k$ quel que soit $z \in \mathrm{t}^{c}$. Par conséquent, la formule ci-dessus donne l'expréssion $(3,9)$ de la forme $\varphi k$. Il en résulte facilement que la 
condition du lemme est suffisant p pour que $\zeta \in \mathscr{E}_{I}^{(q)}(X)$. Supposons maintenant que $\varphi \in \mathscr{C}_{1}^{(q)}(X)$. Alors, $\varphi k=\varphi$ et, d'après l'unicité de $(3,9), \varphi_{j_{1} \cdots j_{1}}^{(q-r)} k=\varphi_{j}^{\left(q-j_{1}\right.}$, pour tout $r=0, \ldots, q, 1 \leqq j_{1}<\cdots<j_{r} \leqq 2 n$, quel que soit $k \in K$. Les formes $\varphi_{j_{1} \cdots j_{r}}^{(q-r)}$ sont donc invariantes par les opérations du groupe $K$ sur $X$; en particulier, elles sont invariantes par les opérations du groupe $T$ dans $X$, lesquelles étant induites par les opérations du groupe $\hat{B} \subset K$. Comme $i(z) \varphi_{j_{1} \cdots j_{r}}^{(q-r)}=0$ quel que soit $z \in 1^{c}$, il en résulte qu'il existe des formes $\psi_{j_{1} \ldots j_{r}}^{(q-r)} \in \mathscr{E}^{(q-r)}(\hat{X})$ telles que $\varphi_{j_{1} \ldots j_{r}}^{(q-r)}=\psi_{j_{1} \cdots j_{r}}^{(q-r)} \hat{p}\left(r=0, \ldots, q, 1 \leqq i_{1}<\cdots<j_{r} \leqq 2 n\right)$. Il est alors clair que $\psi_{j_{1} \cdots j_{r}}^{(q-r)} \in \mathscr{E}_{1}^{(q-r)}(\hat{X})$. La condition est donc nécessaire. Le Lemme 5 est ainsi démontré.

On considère maintenant l'algèbre de $d^{\prime \prime}$-cohomologie $H(X)=\sum_{y \geqq 0} H^{(q)}(X)$ de la variété $X$, c'est-à-dire celle de l'algèbre graduée $\mathscr{E}(X)=\sum_{q \geqq 0} \mathscr{E}^{\left(q^{\prime}\right.}(X)$ munie de l'opérateur différentiel $d^{\prime \prime}$. Les opérations de $K$ dans $\mathscr{E}(X)$ commutant avec $d^{\prime \prime}, d^{\prime \prime}$ applique la sous-algèbre graduée $\mathscr{E}_{1}(X)=\sum_{l \geq 0} \mathscr{E}_{I}^{\prime a}(X)$ en elle-même; soit $H_{l}(X)=\sum_{q \geqq 0} H_{l}^{(q)}(X)$ l'algèbre de $d^{\prime \prime}$-cohomologie de cette sous-algèbre.

Lemme 6. L'homomorphisme de $H_{l}(X)$ dans $H(X)$ défini par l'iniection de $\mathscr{C}_{I}(X)$ dans $\mathscr{E}(X)$ est injectif.

En effet, pour $\varphi \in \mathscr{H}(X)$, soit $\int_{K} \varphi$ la forme que l'on obtient par l'intégration des transformées de $\varphi$ par les opérations de $K$ par rapport à la mesure invariante sur $K$ de volume total $1[5]$. Pour que $\varphi \in \mathscr{C}_{1}(X)$, il faut et il suffit que $\varphi=\int_{K} \varphi$. D'autre part, puisque les opérations de $K$ conservent le type de $\varphi$ et que $d \int_{K} \varphi=\int_{K} d \varphi$, on a $d^{\prime \prime} \int_{K} \varphi=\int_{K} d^{\prime \prime} \varphi$. Par conséquent, si $\varphi \in \mathscr{E}_{1}(X)$ et si il existe une forme $\varphi^{\prime} \in \mathscr{E}(X)$ telle que $\varphi=d^{\prime \prime} \varphi^{\prime}$, on a $\varphi=d^{\prime \prime} \int_{K} \varphi^{\prime}$. Le lemme en résulte.

Lemme 7. Soit $\Omega$ une forme de type $(1,1)$ sur $X$ invariante par $K$ à valeurs dans un espace vectoriel complexe $C^{m}$ de dimension $m$. Si il existe une forme $\xi$ sur $X$, ̀̀ valeurs dans $C^{m}$, telle que $\Omega=d^{\prime \prime} \xi$, alors, il existe une forme $\varphi$ de type. $(1,0)$ sur $X$, ̀̀ valeurs dans $C^{m}$, telle que $\Omega=d \varphi$.

Démonstration. Il suffit de démontrer le lemme dans le cas où $m=1$. D'après le Lemme 6, si $Q \in \mathscr{E}_{l}^{(2)}(X)$ et si $\Omega=d^{\prime \prime} \xi$ avec $\xi \in \mathscr{C}^{(1)}(X)$, il existe une forme $\varphi \in \mathscr{E}_{I}^{(1)}(X)$ telle que $\Omega=d^{\prime \prime} . \subseteq$; si $\Omega$ est de type $(1,1)$, $\subseteq$ est de type $(1,0)$. 
D'après le Lemme 5 , il existe alors une forme $\psi \in \mathscr{C}_{1}^{(1)}(\hat{X})$ et des formes $c_{1}, \ldots$, $c_{2 n} \in \mathscr{C}_{1}^{(0)}(\hat{X})$ telles que

$$
\varphi=\psi \hat{p}+\sum_{j=1}^{2 n}\left(c_{j} \hat{p}\right) \wedge \omega^{j}
$$

D'après le Lemme $4, \psi=0$; il est clair que $c_{1}, \ldots, c_{2 n}$ sont des fonctions constantes. Par conséquent, on a :

$$
\varphi=\sum_{j=1}^{2 n} c_{j} \omega^{j}
$$

La composante de type $(0,1)$ de $\sum_{j=1}^{2 n} c_{j} \omega^{j}$ est égale à $\sum_{j=n+1}^{2 n} c_{j} \omega^{j}$, puisque les formes $\omega^{1}, \ldots, \omega^{n}$ (resp. $\left.\omega^{n+1}, \ldots, \omega^{2 n}\right)$ sont de type $(1,0)$ (resp. de type $\left.(0,1)\right)$. La forme $\varphi$ étant de type $(1,0)$, on a donc:

$$
\varphi=\sum_{i=1}^{n} c_{i} \omega^{i}
$$

Compte tenu de $(3,8)$, il en résulte que

$$
\Omega=d^{\prime \prime} \varphi=\sum_{i=1}^{n} c_{i} d^{\prime \prime} \omega^{i}=\sum_{i=1}^{n} c_{i} d \omega^{i}=d \varphi
$$

ce qui démontre le Lemme 7 .

Démonstration du Théorème 5. D'après le Théorème 4, l'espace fibré $P$ admet une connexion dont la forme $\omega$ est de type $(1,0)$ et dont la forme de courbure $\Omega$ est une forme de type $(1,1)$ invariante par $K$. Si il existe une forme de connexion holomorphe $\omega^{\prime}$ sur $P$, on a $\omega-\omega^{\prime}=\xi p$ où $\xi$ est une forme de type $(1,0)$ sur $X$ à valeurs dans a. Alors, $d \omega-d \omega^{\prime}=d \xi p$. Puisque $d \omega^{\prime}$ est de type $(2,0)$ et que $d \omega=\Omega p$ est de type $(1,1)$, il en résulte que $\Omega=d^{\prime \prime} \xi$. Appliquant le Lemme 7 , on peut trouver une forme $\varphi$ de type $(1,0)$ sur $X$, à valeurs dans a, telle que $\Omega=d \varphi$. La forme $\omega_{0}=\omega-\varphi p$ est une forme de connexion de type $(1,0)$ sur $P$ et $d \omega_{0}=(\Omega-d \varphi) p=0$, ce qui montre que (1) définit une connexion holomorphe intégrable. La base $X$ de $P$ étant simplement connexe, $P$ est alors un espace fibré trivial [1]. Le Théorème 5 est ainsi dẻmontré.

\section{BIBLIOGRAPHIE}

[1] M. F. Atiyah, Complex analytic connections in fibre bundles, Trans. Amer. Math. Soc: 85 (1957), pp. 181-207.

[2] A. Borel, Kahlerian coset spaces of semi-simple Lie groups, Proc. Nat. Acad. Sci. U.S.A. 
40 (1954), pp. 1147-1151.

[ 3 ] R. Bott, Homogenous vector bundles, Ann. of Math. 66 (1957), pp. 203-248.

[4] H. Cartan, Notions d'algèbre différentielle; applications aux groupes de Lie et aux variétés oû opère un groupe de Lie, C.B.R.M. Colloque de Topologie (Espaces fibrés), Bruxelles, 1951.

[5] C. Chevalley-S. Eilenberg, Cohomology theory of Lie groups and Lie algebras, Trans. Amer. Math. Soc. 63 (1948), pp. 85-124.

[6] F. Hirzebruch, Neue topologische Methoden in der algebraischen Geometrie, Ergibnisse der Mathematik und ihrer Grensgebiete, Heft 9, Berlin, 1956.

[ 7 ] J.-L. Koszul, Multiplicateurs et classes caracteristiques, Trans. Amer. Math. Soc. 89 (1958), pp. 256-266.

[ 8 ] J.-L. Koszul, Espaces fibrés associés et préassocics, à paraître dans Nagoya Math. J. 15 (1959).

[9] D. Montgomery, Simply connected homogeneous spaces, Proc. Amer. Math. Soc. 1 (1950), pp. 467-469.

[10] A. Morimoto, Sur le groupe d'automorphismes d'un espace fibré principal complexe analytique, Nagoya Math. J. 13 (1958), pp. 157-178.

[11] K. Nomizu, Lie groups and differential geometry, Publication of Japan Math. Soc. No. 2, Tokyo, 1956.

[12] H. C. Wang, Closed manifolds with homogeneous complex structure, Amer. J. of Math. 76 (1954), pp. 1-32.

[13] Séminaire Sophus Lie, E.N.S. 1954-1955, Paris, 1955.

Strasbourg, France et Osaka, Japon 